\title{
HIERARCHIES OF EFFECTIVE DESCRIPTIVE SET THEORY
}

\author{
BY \\ PETER G. HINMAN $\left({ }^{1}\right)$
}

1. Introduction and summary. The theory of hierarchies deals with the classification of objects according to some measure of their complexity. Such classifications have been fruitful in several areas of mathematics: analysis (descriptive set theory), recursion theory, and the theory of models. Although much of the hierarchy theory of each of these areas was developed independently of the others, Addison, in the series of papers [Ad 1-6], has shown not only that there are deep-seated analogies among these theories, but that indeed many of their results can be derived from those of a general theory of hierarchies. Toward a further consolidation of these theories, this paper will study the relationships and analogies between certain classical hierarchies of descriptive set theory and their counterparts in recursion theory.

The roots of modern hierarchy theory lie in the investigations of Baire, Borel, Lebesgue, and others around the turn of the century. As analysts with a concern for the foundations of their subject, they felt that constructions effected by means of the axiom of choice or the set of all countable ordinals were less secure than those carried out by more elementary means. They sought to discover what role these suspect constructions played in analysis and whether or not they could be avoided altogether. Thus descriptive set theory arose with the goal of identifying, classifying, and studying those sets (of real numbers) which were of interest for analysis and for which an "explicit" construction could be given. Needless to say, there was vigorous disagreement as to just what constituted an explicit construction.

The first large class of sets studied were the Borel sets. Since each Borel set can be constructed by iteration of the elementary operations of countable union and complementation over a countable well-ordering, it was in general agreed that these sets are constructively defined. Lebesgue defined in 1905 [Le] a set outside of the Borel classification, but although it avoided use of the axiom of choice, Lebesgue's construction used the set of countable ordinals and hence was rejected by some (see, for example, [Bo, p. 208]).

In 1917, Suslin discovered the operation $\mathscr{A}$ (for analytic-see definition below)

Received by the editors December 8, 1967 and, in revised form, October 25, 1968.

( ${ }^{1}$ Some of this material appears in quite different form in the author's doctoral dissertation [Hi]. We want to thank Professor J. W. Addison, Jr. for directing us to these questions and for the many stimulating conversations which made essential contributions to this work. Parts of our research were supported by the National Science Foundation. 
and showed that the class of sets (analytic sets) obtainable by a single application of $\mathscr{A}$ to families of intervals properly included the class of Borel sets-in fact, that the Borel sets comprise just those analytic sets whose complements are also analytic. Suslin's discovery had also great methodological significance as it led directly to the abstract notion of a set operation and the recognition that many such operations are sufficiently "effective" to provide an explicit construction for each member of the inductive closure of the class of intervals under the operation. In particular, the sets obtained by closing under $\mathscr{A}(C$-sets or ensembles criblés) were studied in detail by Selivanovskij [Se].

We now make some definitions in order to describe more precisely the plan of this paper. Let $N$ be the set of natural numbers and $N^{N}$ the set of functions from $N$ into $N$. As is customary in modern descriptive set theory we shall replace the reals by $N^{N}$ (homeomorphic to the irrationals). Letters $a, b, \ldots, z$ will serve as variables over $N$ and $\alpha, \beta, \ldots, \varepsilon$ as variables over $N^{N}$. For any set $A, \mathscr{P}(A)$ is the power set of $A$. A function $F \in \mathscr{P}(A)^{N}$ is called an indexed family of subsets of $A$. An ( $N$-ary) operation over $A$ is a function $\Gamma: \mathscr{P}(A)^{N} \rightarrow \mathscr{P}(A)$. For example,

$$
\begin{aligned}
\cup(F) & =\{a / \exists p[a \in F(p)]\} ; \\
\mathscr{A}(F) & =\{a / \exists \beta \forall y[a \in F(\bar{\beta}(y))]\} ; \\
\lim \inf (F) & =\{a / \exists p \forall q[p<q \rightarrow a \in F(q)]\} .
\end{aligned}
$$

Let $\mathscr{I}$ (the initial class) be any subset of $\mathscr{P}(A)$. The inductive closure of $\mathscr{I}$ under $\Gamma$, denoted by $\mathscr{C} \Gamma(A, \mathscr{I})$, is the smallest class $\mathscr{C} \subseteq \mathscr{P}(A)$ such that:

(i) $\mathscr{I} \subseteq \mathscr{C}$;

(ii) $F \in \mathscr{C}^{N} \rightarrow \Gamma(F) \in \mathscr{C}$;

(iii) $C \in \mathscr{C} \rightarrow(A \sim C) \in \mathscr{C}$.

When $A=N^{N}$ and $\mathscr{I}$ is the class of intervals, we write simply $\mathscr{C} \Gamma$ for $\mathscr{C} \Gamma(A, \mathscr{I})$. Then the class of Borel sets is $\mathscr{C} \cup$ and the class of $C$-sets is $\mathscr{C} \mathscr{A}$.

Our first results were motivated by the similarities we noticed between this definition and Kleene's definition of recursiveness relative to a (type-2) functional [KI 3]. The stage was set by two characterizations of the hyperarithmetic subsets of $N$ : as those recursive in the functional ${ }^{2} E[\mathbf{K l ~ 3 , ~ p . ~ 4 8 ] ~ a n d ~ a s ~ t h o s e ~ e n c o m p a s s e d ~ b y ~}$ an "effective" version of the Borel hierarchy [Ad 1, 2]. In $\$ 2$ we generalize Addison's construction to define for each operation $\Gamma$ a class $\mathscr{C} \Gamma(=\mathscr{C} \Gamma(N, \mathscr{I}))$, the effective inductive closure of the class of singletons under $\Gamma$. Roughly, this is done by assigning to each set $C$ as it is generated an index $\iota(C)$ and at each stage of the inductive definition applying $\Gamma$ only to those families $F$ such that $\lambda p[\iota(F(p))]$ is recursive in some set previously generated. Also with each $\Gamma$ we associate a functional $g_{\Gamma}$ (such that $g_{U}={ }^{2} E$ ) and prove that for a large class of operations $\Gamma, \mathscr{C}^{\Gamma}$ is exactly the class of sets recursive in $g_{\Gamma}$.

Since an analytic set is obtained by a single application of $\mathscr{A}$, by analogy with a common notation it seems natural to denote the class of analytic sets by $\boldsymbol{\Sigma}_{1}^{\mathscr{A}}$. 
Consistent use of this notation gives, for example, $\boldsymbol{\Sigma}_{1}^{\cup}$ for the open sets and $\boldsymbol{\Pi}_{2}^{\cup}$ for the $G_{\delta}$-sets. Then Suslin's theorem is: $\mathscr{C}^{\cup}=\Delta_{1}^{\mathscr{A}}$. In the 1920 's (but not published until later; see [Ka-Li]) Kolmogorov, abstracting from the relationship $\cup: \mathscr{A}$, defined the $R$-operator, which maps operations to operations and satisfies $R \cup=\mathscr{A}$, and $\mathscr{C} \Gamma=\Delta_{1}^{R \Gamma}$ was proposed as a generalization of Suslin's theorem. The history of this conjecture is unclear, but its status seems to have been unknown until 1953 and the publication of $[\mathbf{L j} \mathbf{1}, \mathbf{2}]$. In these papers, Ljapunov shows that the conjecture as stated fails for a rather trivial reason (cf. the proof of 4.7 below), but that a closely related operator $R^{*}$ satisfies: $\mathscr{C}^{\Gamma} \subseteq \Delta_{1}^{R^{*} \Gamma}$. Ljapunov studied the sequence of operations $\mathscr{R}^{v}: \mathscr{R}^{0}=U, \mathscr{R}^{v+1}=R^{*} \mathscr{R}^{v}$, and for limit $\lambda, \mathscr{R}^{\lambda}$ is a "join" of $\left\{\mathscr{R}^{\xi} / \xi<\lambda\right\}$, and showed that at every level above the first, $\mathscr{C}^{\mathscr{R}^{v}}$ is a proper subset of $\Delta_{1}^{\mathscr{R}^{v+1}}$. Hence the problem of finding a direct generalization of Suslin's theorem is still open.

We felt for a long time that the effective situation might well be different. The proof that $\mathscr{C}^{\Gamma} \subseteq \Delta_{1}^{R^{*} \Gamma}$ offers mainly combinatorial difficulty, and together with the basic properties of $R$ and $R^{*}$ is given in $\S \S 3,4$ below. Since $R^{*} U$ is equivalent to $\mathscr{A}$, it follows from Addison's results and those of [K1 2] that the converse inclusion holds for $\Gamma=U$. We conjectured in [Hi] that this was so in general. Among other reasons, it seemed to us that Ljapunov's proof depended essentially on the presence of $N^{N}$ rather than $N$ as the base space and thus could not be carried over to the effective case. An example of this phenomenon is studied in [Be], where it is shown that although $\mathscr{C} \cup\left(N^{N}, \boldsymbol{\Sigma}_{1}^{\mathscr{A}}\right)$ is a proper subset of $\Delta_{2}^{\mathscr{A}}[\mathbf{K u}]$, the corresponding effective classes over $N$ are equal. The conjecture is especially tempting in that the properties of $\Pi_{1}^{R^{\bullet} \Gamma}$ are very similar to those of $\Pi_{1}^{\mathscr{A}}\left(=\Pi_{1}^{1}\right)$. In particular, each set in $\Pi_{1}^{R^{*} \Gamma}$ is a transfinite union of a sequence of sets (its constituents) which are definable by an inductive procedure based on $\Gamma$. This is explained in $\$ 6$.

The facts, however, are otherwise, and it turns out that this inductive procedure differs in a subtle way from that used to define $\mathscr{C}^{\Gamma}$. In $\S 5$ we develop the theory of a functional $g_{\Gamma}^{\#}$, which differs from $g_{\Gamma}$ in including in its domain some partial functions from $N$ into $N$. Under a suitable extension of Kleene's notion of recursiveness to include functionals of this type, we show in $\S 7$ that $\Delta_{1}^{R^{*} \Gamma}$ is exactly the class of sets recursive in $g_{\Gamma}^{\#}$ and that for $\Gamma$ which are as powerful as $\mathscr{A}$-in particular, if $\Gamma$ is $\mathscr{R}^{\nu}$ with $\nu \geqq 1$-this class includes sets not recursive in $g_{\Gamma}$. Thus the proposed generalization of Suslin's theorem fails in the effective case as well.

Finally in $\S 8$ we discuss briefly the theory of the effective hierarchies over $N^{N}$. We are not able to extend the results of $\$ 2$ to this case. Not only does there seem to be no natural definition for $\mathscr{C} \Gamma\left(N^{N}, \mathscr{I}\right)$, but for no known definition does this class exhaust the sets of functions recursive in $g_{\Gamma}$. On the other hand, the relationship between $R^{*} \Gamma$ and $g_{\Gamma}^{\#}$ remains the same when functions are allowed as arguments. In particular, it follows that $g_{R^{*} \Gamma}$ is of the same degree as the diagonalization or superjump of $g_{\Gamma}^{\#}$.

These results leave open a number of interesting questions. It seems likely, for example, that the result just mentioned could be extended to show that $g_{R^{*} \Gamma}^{\#}$ is also 
of the same degree as the superjump of $g_{\Gamma}^{\#}$, where "degree" is redefined to require that all partial functions be admitted as arguments. In any case, $R$ behaves much like a jump operator on operations; the $\mathscr{R}^{v}$ correspond to Kleene's sets $H_{a}(a \in \mathcal{O})$. Appropriate restriction of the join operation at limit ordinals should lead to an interesting class of operations. Plausible conjectures are that the sets generated by these operations coincide with the ramified analytic sets or those recursive in the (type-3) superjump functional.

Most of our notation will follow either [KI 1] or [Kl 3] with which we assume familiarity. In particular, our indices of partial recursive functions are those of [KI 3]. To assert that an index $a$ defines a computation for some list of arguments $\mathfrak{A}$ we write equivalently " $\{a\}(\mathfrak{U}) \downarrow "$ or " $\mathfrak{A} \in \operatorname{Dm}\{a\} "$. The statement $\{a\}(\mathfrak{U}) \in X$ is true if and only if $\mathfrak{A} \in \operatorname{Dm}\{a\}$ and the value $\{a\}(\mathfrak{A})$ belongs to $X$. We shall assume some fixed 1-1 correspondence between the set of finite sequences of natural numbers and $N$, but we shall never indicate it explicitly. Thus $\left\langle a_{0}, \ldots, a_{m-1}\right\rangle$ denotes both a sequence and its corresponding number. If $s=\left\langle a_{0}, \ldots, a_{m-1}\right\rangle$ and $t=\left\langle b_{0}, \ldots, b_{n-1}\right\rangle$, then $\ln (s)=m, s\langle p\rangle=\left\langle a_{0}, \ldots, a_{m-1}, p\right\rangle,\langle p\rangle s=\left\langle p, a_{0}, \ldots, a_{m-1}\right\rangle$ $s * t=\left\langle a_{0}, \ldots, a_{m-1}, b_{0}, \ldots, b_{n-1}\right\rangle,(s)_{i}=\left(a_{i}\right.$, if $i<m ; 0$, otherwise $)$, and $s \subseteq t$ if and only if $m \leqq n$ and for $i<m, a_{i}=b_{i}$. We often write $\{a\}(s)$ to mean $\{a\}\left(a_{0}, \ldots, a_{m-1}\right)$. For $i \leqq \ln (s), s\left|i=\left\langle a_{0}, \ldots, a_{i-1}\right\rangle . s\right| 0=\varnothing$, the empty sequence.

\section{The effective hierarchies.}

Definition 2.1. For any $N$-ary operation $\Gamma$ over $N$ :

(a) $I^{\Gamma}$ is the smallest set such that for any $a, m, u$, and $v$ :

(i) $\langle 0, m\rangle \in I^{\Gamma}$;

(ii) $u \in I^{\Gamma}$ and $\forall p\left[\{a\}^{[u: \Gamma]}(p) \in I^{\Gamma}\right] \rightarrow\langle 1, u, a\rangle \in I^{\Gamma}$;

(iii) $v \in I^{\Gamma} \rightarrow\langle 2, v\rangle \in I^{\Gamma}$;

(b) for any $m$ and any $\langle 1, u, a\rangle$ and $\langle 2, v\rangle \in I^{\Gamma}$ :

$[\langle 0, m\rangle ; \Gamma]=\{n / n=m\}$;

$[\langle 1, u, a\rangle ; \Gamma]=\Gamma\left(\lambda p\left[\{a\}^{[u ; \Gamma]}(p) ; \Gamma\right]\right)$;

$[\langle 2, v\rangle ; \Gamma]=N \sim[v ; \Gamma]$

(c) $\mathscr{C}^{\Gamma}=\left\{[u ; \Gamma] / u \in I^{\Gamma}\right\}$;

(d) for $u \in I^{\Gamma},|u|^{\Gamma}$ is an ordinal as follows:

$|\langle 0, m\rangle|^{\Gamma}=0$;

$|\langle 1, u, a\rangle|^{\Gamma}=\max \left\{|u|^{\Gamma}+1, \sup _{p}\left\{\left|\{a\}^{[u: \Gamma]}(p)\right|^{\Gamma}+1\right\}\right\} ;$

$|\langle 2, v\rangle|^{\Gamma}=|v|^{\Gamma}$

(e) for any ordinal $\nu, I_{v}^{\Gamma}=\left\{u \mid u \in I^{\Gamma}\right.$ and $\left.|u|^{\Gamma} \leqq \nu\right\}$, and

$\Sigma_{v}^{\Gamma}=\left\{[\langle 1, u, a\rangle ; \Gamma] /\langle 1, u, a\rangle \in I_{v}^{\Gamma}\right\} ;$

$\Pi_{v}^{\Gamma}=\left\{[\langle 2,\langle 1, u, a\rangle\rangle ; \Gamma] /\langle 1, u, a\rangle \in I_{v}^{\Gamma}\right\} ;$

$\Delta_{v}^{\Gamma}=\Sigma_{v}^{\Gamma} \cap \Pi_{v}^{\Gamma}$.

Definition 2.2. For any set $A$ and any $N$-ary operation $\Gamma$ over $A, \Gamma$ is analytic if and only if for any $F, G \in \mathscr{P}(A)^{N}$ and any $a, b \in A$, if $\forall p(a \in F(p) \leftrightarrow b \in G(p))$, then $a \in \Gamma(F) \leftrightarrow b \in \Gamma(G)$. 
The examples of $\S 1$ are all analytic. An example of a nonanalytic operation over $N$ is:

$$
\Gamma(F)=\{m / \forall p n(n<m \wedge m \in F(p) \rightarrow n \in F(p))\} .
$$

Intuitively, the distinguishing feature of an analytic operation is that the question of whether or not $a \in \Gamma(F)$ depends only on $\{p / a \in F(p)\}$ and not on any conditions involving any $b$ different from $a$. This principle is formalized in the second conclusion of the following lemma.

LEMMA 2.3. For any sets $A$ and $C$ and any analytic $N$-ary operation $\Gamma$ over $A$, let $\Lambda$ be the $N$-ary operation over $C$ defined as follows. For any $G \in \mathscr{P}(C)^{N}$, any $c \in C$, and any $p \in N$, let $G^{c}(p)=(A$, if $c \in G(p) ; \varnothing$, otherwise $)$ and set $\Lambda(G)=\left\{c \mid \Gamma\left(G^{c}\right)=A\right\}$. Then $\Lambda$ is analytic over $C$. In particular, if $A=C$, then $\Lambda=\Gamma$ so for any $G \in \mathscr{P}(A)^{N}$ and any $a$,

$$
a \in \Gamma(G) \leftrightarrow \Gamma\left(G^{a}\right)=A .
$$

Proof. Suppose $G_{1}$ and $G_{2}$ in $\mathscr{P}(C)^{N}$ are such that $\forall p\left(c \in G_{1}(p) \leftrightarrow d \in G_{2}(p)\right)$. Then $G_{1}^{c}=G_{2}^{d}$, so

$$
c \in \Lambda\left(G_{1}\right) \leftrightarrow \Gamma\left(G_{1}^{c}\right)=A \leftrightarrow \Gamma\left(G_{2}^{d}\right)=A \leftrightarrow d \in \Lambda\left(G_{2}\right) .
$$

Hence $\Lambda$ is analytic. For the second part, let $G$ be in $\mathscr{P}(A)^{N}$ and $a \in A$. Then for any $b \in A, \forall p\left(a \in G(p) \leftrightarrow b \in G^{a}(p)\right)$, and hence by the analyticity of $\Gamma, a \in \Gamma(G)$ $\leftrightarrow b \in \Gamma\left(G^{a}\right)$. Since then $b$ is independent of $a, a \in \Gamma(G) \leftrightarrow \Gamma\left(G^{a}\right)=A \leftrightarrow a \in \Lambda(G)$.

Henceforth we shall use the same symbol $\Gamma$ to denote an analytic operation together with all its extensions defined as in the lemma. Then the defining property of 2.2 holds also when $F$ and $G$ are families over different sets.

Definition 2.4. For any analytic operation $\Gamma$, any $\alpha \in N^{N}$, and any $p \in N$, let $G^{\alpha}(p)=(N$, if $\alpha(p)=0 ; \varnothing$, otherwise $)$.

$$
\begin{aligned}
g_{\Gamma}(\alpha) & =0, & & \text { if } \Gamma\left(G^{\alpha}\right)=N ; \\
& =1, & & \text { otherwise. }
\end{aligned}
$$

EXAMPLES. $g_{\cup}(\alpha)=0 \leftrightarrow \exists p(\alpha(p)=0) ; g_{\mathscr{A}}(\alpha)=0 \leftrightarrow \exists \beta \forall x(\alpha(\bar{\beta}(x))=0)$.

LEMMA 2.5. For any analytic operation $\Gamma$ :

(a) for any $F \in \mathscr{P}(N)^{N}$, any $m \in N$, and any $\alpha \in N^{N}$, if $\forall p(\alpha(p)=0 \leftrightarrow m \in F(p))$, then $g_{\Gamma}(\alpha)=0 \leftrightarrow m \in \Gamma(F)$;

(b) if for all $u \in I^{\Gamma}$ we define by recursion a function $\llbracket u$; $\Gamma \rrbracket$ by:

$$
\begin{aligned}
\llbracket\langle 0, m\rangle ; \Gamma \rrbracket & =\lambda n(0, \text { if } m=n ; 1, \text { otherwise }) ; \\
\mathbb{L}\langle 1, u, a\rangle ; \Gamma \rrbracket & =\lambda m\left(g_{\Gamma}\left(\lambda p \llbracket\{a\}^{[u ; \Gamma]}(p) ; \Gamma \rrbracket(m)\right)\right) ; \\
\mathbb{L}\langle 2, v\rangle ; \Gamma \rrbracket & =\lambda m(1-\llbracket v ; \Gamma \rrbracket(m)) ;
\end{aligned}
$$

then $\llbracket u ; \Gamma \rrbracket$ is the characteristic function of $[u ; \Gamma]$.

Proof. If $F, m$ and $\alpha$ satisfy the hypothesis of (a), then $G^{\alpha}=F^{m}$, that is, $\forall p\left(G^{\alpha}(p)\right.$ $=N \leftrightarrow m \in F(p))$. It follows by Lemma 2.3 that

$$
m \in \Gamma(F) \leftrightarrow \Gamma\left(G^{\alpha}\right)=N \leftrightarrow g_{\Gamma}(\alpha)=0 .
$$


Part (b) follows immediately by induction over $I^{\Gamma}$.

COROllary 2.6. For any analytic operation $\Gamma, \mathscr{C}^{\Gamma} \subseteq_{1} \mathrm{sc}\left(g_{\Gamma}\right)$. In fact, there exists a primitive recursive function $\pi$ such that for all $u \in I^{\Gamma}, \llbracket u ; \Gamma \rrbracket=\lambda m\{\pi(u)\}\left(g_{\Gamma}, m\right)$.

Proof. Let $\phi$ be a partial recursive function defined by cases as follows: for any functional $f$ and any $m, n, a, e, u, v$,

(i) $\phi(f,\langle 0, m\rangle, n, e)=(0$, if $n=m$; 1 , otherwise $)$;

(ii) $\phi(f,\langle 1, u, a\rangle, m, e) \cong f\left(\lambda p\{e\}\left(f,\{a\}^{\lambda n\{e\}(f, u, n)}(p), m\right)\right)$;

(iii) $\phi(f,\langle 2, v\rangle, m, e) \cong 1-\{e\}(f, v, m)$;

(iv) $\phi(f, u, m, e)$ is undefined otherwise.

By the recursion theorem there exists an index $e_{0}$ such that

$$
\phi\left(f, u, m, e_{0}\right) \cong\left\{e_{0}\right\}(f, u, m) .
$$

Let $\pi(u)$ be an index for $\lambda f m\left\{e_{0}\right\}(f, u, m)$-that is,

$$
\{\pi(u)\}(f, m) \cong\left\{e_{0}\right\}(f, u, m) .
$$

Then it is routine to check by induction on $I^{\Gamma}$ that $\pi$ has the required property. The first statement of the corollary then follows immediately by 2.5 .

The converse of Corollary 2.6 is certainly not true for every analytic $\Gamma$. For example, if $\Gamma(F)=\varnothing$ for all $F$, then $\mathscr{C}^{\Gamma}$ consists only of $N, \varnothing$, the singletons, and their complements, while ${ }_{1} \mathrm{sc}\left(g_{\Gamma}\right)$ is the set of recursive sets. Even for less trivial operations it is by no means obvious that $\mathscr{C}^{\Gamma}$ is closed under "recursive in". Roughly, the difficulty is that too much is concentrated in the main inductive clause of Definition 2.1(b). Recursions in previously generated sets, diagonalization, and application of $\Gamma$ are all accomplished at one fell swoop. While this seems appropriate as an effective counterpart of the inductive clause in the definition of $\mathscr{C} \Gamma$, it may generate pathologies for certain operations.

The following definition and theorem were motivated by these considerations and some closely related results of Moschovakis [Mo 1]. They will greatly facilitate the proof of Theorem 2.10 below and may also be of some independent interest.

Definition 2.7. For any functional $f$ :

(a) $J^{f}$ is the smallest set such that for any $a$ and $v$ :

(i) $\langle 3\rangle \in J^{f}$;

(ii) $v \in J^{f}$ and $\operatorname{Dm}\{a\}^{[v: f]}=N \rightarrow\langle 4, v, a\rangle \in J^{f}$;

(iii) $\forall m\left(\llbracket v ; f \rrbracket(m) \in J^{f}\right) \rightarrow\langle 5, v\rangle \in J^{f}$;

(iv) $v \in J^{f} \rightarrow\langle 6, v\rangle \in J^{f}$;

(b) for any $\langle 4, v, a\rangle,\langle 5, v\rangle$, and $\langle 6, v\rangle \in J^{f}$ :

$\llbracket\langle 3\rangle ; f \rrbracket=\lambda m(0$, if $m=0 ; 1$, otherwise $)$;

$\llbracket\langle 4, v, a\rangle ; f \rrbracket=\{a\}^{\llbracket v ; f]}$;

$\llbracket\langle 5, v\rangle ; f \rrbracket=\lambda m \llbracket \llbracket v ; f \rrbracket(m) ; f \rrbracket(m) ;$

$\llbracket\langle 6, v\rangle ; f \rrbracket=\lambda m f(\llbracket v ; f \rrbracket) ;$

(c) $\mathscr{D}^{f}=\left\{\llbracket v ; f \rrbracket / v \in J^{f}\right\}$. 
THEOREM 2.8. For any functional $f, \mathscr{D}^{f}={ }_{1} \mathrm{sc}(f)$. In fact, there exist primitive recursive functions $\rho$ and $\sigma$ such that:

(a) for any $v \in J^{f}$, $v ; f \rrbracket=\{\rho(v)\}^{f}$;

(b) for any a such that $\operatorname{Dm}\{a\}^{f}=N, \sigma(a) \in J^{f}$ and $\{a\}^{f}=\llbracket \sigma(a) ; f \rrbracket$.

Proof. The construction of $\rho$ is very similar to that of $\pi$ in the proof of Corollary 2.6 and will be omitted. For (b) we construct a partial recursive function $\psi$ such that:

$$
x \in \operatorname{Dm}\{a\}^{f} \rightarrow \psi(a, x) \in J^{f} \text { and } \llbracket \psi(a, x) ; f \rrbracket=\lambda z\{a\}^{f}(x)
$$

Given such a $\psi$, we define $\sigma$ from it as follows. Since $f$ is fixed, we write simply $\llbracket v \rrbracket$ for $\llbracket v ; f \rrbracket$. Let $\sigma_{1}$ be a primitive recursive function such that for any $\alpha,\left\{\sigma_{1}(a)\right\}^{\alpha}(x)$ $\cong \psi(a, x)$, and take $\sigma(a)=\left\langle 5,\left\langle 4,\langle 3\rangle, \sigma_{1}(a)\right\rangle\right\rangle$. Then for any $a$ such that $\operatorname{Dm}\{a\}^{f}=N$,

$$
\begin{aligned}
\llbracket \sigma(a) \rrbracket(x) & =\llbracket\left[\llbracket 4,\langle 3\rangle, \sigma_{1}(a)\right\rangle \rrbracket(x) \rrbracket(x) \\
& =\llbracket\left\{\sigma_{1}(a)\right\}^{\langle 3\rangle} \rrbracket(x) \rrbracket(x)=\llbracket \psi(a, x) \rrbracket(x)=\{a\}^{f}(x) .
\end{aligned}
$$

We define $\psi$ via the recursion theorem and a partial recursive function $\phi$ defined by cases. If $e_{0}$ is an index such that $\phi\left(a, x, e_{0}\right) \cong\left\{e_{0}\right\}(a, x)$ and $\psi=\left\{e_{0}\right\}$, then it is easy to prove by induction on computations $[\mathbf{K I} 3,3.8]$ that $\psi$ has the property $\left(\mathrm{b}^{\prime}\right)$. We give parts of this proof along with the definition of $\phi$.

(i) If $(a)_{0}=0$, let $\sigma_{2}$ be a primitive recursive function such that for any $\alpha, x, e$, and $y$,

$$
\left\{\sigma_{2}(a, x, e)\right\}^{\alpha}(y) \cong\{e\}\left((a)_{3},\langle y\rangle x\right),
$$

and set $\phi(a, x, e)=\left\langle 6,\left\langle 5,\left\langle 4,\langle 3\rangle, \sigma_{2}(a, x, e)\right\rangle\right\rangle\right\rangle$.

$$
\begin{aligned}
\llbracket \psi(a, x) \rrbracket(z) & =\llbracket \phi\left(a, x, e_{0}\right) \rrbracket(z) \\
& =f\left(\lambda y \llbracket \llbracket\left\langle 4,\langle 3\rangle, \sigma_{2}\left(a, x, e_{0}\right)\right\rangle \rrbracket(y) \rrbracket(y)\right) \\
& =f\left(\lambda y \llbracket\left\{\sigma_{2}\left(a, x, e_{0}\right)\right\}^{<3\rangle}(y) \rrbracket(y)\right) \\
& =f\left(\lambda y \llbracket \psi\left((a)_{3},\langle y\rangle x\right) \rrbracket(y)\right) \\
& =f\left(\lambda y\left\{(a)_{3}\right\}^{f}(\langle y\rangle x)\right) \quad \text { (Induction hypothesis) } \\
& =\{a\}^{f}(x) .
\end{aligned}
$$

(ii) If $(a)_{0}=1,2$, or 3 , let $\sigma_{3}$ be a primitive recursive function such that for any $\alpha, x$, and $z$,

$$
\left\{\sigma_{3}(a, x)\right\}^{\alpha}(z) \cong\{a\}^{f}(x)
$$

and set $\phi(a, x, e)=\left\langle 4,\langle 3\rangle, \sigma_{3}(a, x)\right\rangle$.

(iii) If $(a)_{0}=4$, let $\sigma_{4}$ be a primitive recursive function such that for any $\alpha, x, e$, and $z$,

$$
\left\{\sigma_{4}(a, x, e)\right\}^{\alpha}(z) \cong\{e\}\left((a)_{2},\langle\alpha(z)\rangle x\right)
$$


and set $\phi(a, x, e) \cong\left\langle 5,\left\langle 4,\{e\}\left((a)_{3}, x\right), \sigma_{4}(a, x, e)\right\rangle\right\rangle$.

$$
\begin{aligned}
& \llbracket \psi(a, x) \rrbracket(z)=\llbracket \phi\left(a, x, e_{0}\right) \rrbracket(z) \\
& =\llbracket \llbracket\left\langle 4,\left\{e_{0}\right\}\left((a)_{3}, x\right), \sigma_{4}\left(a, x, e_{0}\right)\right\rangle \rrbracket(z) \rrbracket(z) \\
& =\mathbb{[}\left\{\sigma_{4}\left(a, x, e_{0}\right)\right\}^{\left[\nu\left((a)_{3}, x\right)\right]}(z) \rrbracket(z) \\
& =\llbracket \psi\left((a)_{2},\left\langle\llbracket \psi\left((a)_{3}, x\right) \rrbracket(z)\right\rangle x\right) \rrbracket(z) \\
& =\left\{(a)_{2}\right\}^{f}\left(\left\langle\left\{(a)_{3}\right\}^{f}(x)\right\rangle x\right) \text { (Induction hypothesis) } \\
& =\{a\}^{f}(x) \text {. }
\end{aligned}
$$

(iv) If $(a)_{0}=5$, the treatment is similar to (iii).

(v) If $(a)_{0}=6$, let $x^{\prime}$ be the prescribed rearrangement of the components of $x$ and set $\phi(a, x, e) \cong \phi\left((a)_{4}, x^{\prime}, e\right)$.

(vi) If $(a)_{0}=9$, let $b, y$, and $y^{\prime}$ be such that $x=\langle b\rangle y * y^{\prime}$ and $\{a\}^{f}(x) \cong\{b\}^{f}(y)$, and set $\phi(a, x, e) \cong \phi(b, y, e)$.

Definition 2.9. For any analytic operations $\Gamma$ and $\Lambda, \Lambda$ subsumes $\Gamma$ if and only if there exists a primitive recursive function $\alpha$ such that for any family $F$,

$$
\Gamma(F)=\Lambda(\lambda p F(\alpha(p))) .
$$

EXAMPLES. $\cup(F)=\mathscr{A}\left(\lambda p F\left((p)_{0}\right)\right) . \cap(F)=\mathscr{A}(\lambda p F(\ln (p)))$.

THEOREM 2.10. For any analytic operation $\Gamma$, if $\Gamma$ subsumes $U$, then $\mathscr{C}^{\Gamma}={ }_{1} \mathrm{sc}\left(g_{\Gamma}\right)$. In fact there exists a primitive recursive function $\tau$ such that for any $v \in J^{g_{\Gamma}}, \tau(v) \in I^{\Gamma}$ and $[\tau(v) ; \Gamma]=\left\{m / \llbracket v ; g_{\Gamma} \rrbracket(m)=0\right\}$.

Proof. The second statement together with 2.6 and 2.8 implies the first. Let $\alpha$ be a recursive function by which $\Gamma$ subsumes $U$. We shall construct a partial recursive function $\psi$ such that for any $v \in J^{g_{\Gamma}}, \psi(v) \in I^{\Gamma}$ and

$$
[\psi(v) ; \Gamma]=\left\{\langle m, n\rangle /\left[\llbracket ; g_{\Gamma} \rrbracket(m)=n\right\} .\right.
$$

Given such a $\psi$, we define $\tau$ from it as follows. Let $b$ and $c$ be indices such that for any set $A,\{b\}^{A}$ enumerates $\{\langle 0, m\rangle \mid\langle m, 0\rangle \in A\}$ and $\{c\}^{A}=\lambda p\{b\}^{A}(\alpha(p))$. Let $\tau_{1}$ be a primitive recursive function such that for any $v, q$, and $A,\left\{\tau_{1}(v)\right\}^{A}(q) \cong\langle 1, \psi(v), c\rangle$, and set $\tau(v)=\left\langle 1,\langle 0,0\rangle, \tau_{1}(v)\right\rangle$. Since $\Gamma$ is fixed, we write $[u]$ for $[u ; \Gamma]$. For $v \in J^{g_{\Gamma}}$,

$$
\begin{aligned}
{[\tau(v)] } & =\Gamma\left(\lambda q\left[\left\{\tau_{1}(v)\right\}^{[\langle 0,0\rangle]}(q)\right]\right)=\Gamma\left(\lambda q\left[\left\{\tau_{1}(v)\right\}^{[\langle 0,0\rangle]}(\alpha(q))\right]\right) \\
& =U(\lambda q[\langle 1, \psi(v), c\rangle])=[\langle 1, \psi(v), c\rangle] \\
& =\Gamma\left(\lambda p\left[\{c\}^{[\psi(v)]}(p)\right]\right)=U\left(\lambda p\left[\{b\}^{[v(v)]}(p)\right]\right) \\
& =U\{[\langle 0, m\rangle] /\langle m, 0\rangle \in[\psi(v)]\}=\left\{m /\left[v ; g_{\Gamma} \rrbracket(m)=0\right\} .\right.
\end{aligned}
$$

As usual, $\psi$ is defined via the recursion theorem as $\left\{e_{0}\right\}$, where $\phi\left(v, e_{0}\right) \cong\left\{e_{0}\right\}(v)$ and $\phi$ is defined by cases as follows.

(i) $\phi(\langle 3\rangle, e)=\langle 0,0\rangle$.

(ii) If $v=\langle 4, w, a\rangle$, let $b$ and $c$ be indices such that for any set $A,\{b\}^{A}$ enumerates $\left\{\langle 0,\langle m, n\rangle\rangle \mid\{a\}^{A}(m) \cong n\right\}$ and $\{c\}^{A}=\lambda p\{b\}^{A}(\alpha(p))$, and set 


$$
\begin{aligned}
\phi(v, e) & \cong\langle 1,\{e\}(w), c\rangle . \\
{[\psi(v)] } & =\left[\left\langle 1,\left\{e_{0}\right\}(w), c\right\rangle\right]=\Gamma\left(\lambda p\left[\{c\}^{[w(w)]}(p)\right]\right) \\
& =\cup\left\{[\langle 0,\langle m, n\rangle\rangle] /\{a\}^{[v(w)]}(m) \cong n\right\} \\
& =\left\{\langle m, n\rangle /\{a\}^{\left[w: g_{\Gamma}\right]}(m) \cong n\right\} \quad \text { (Induction hypothesis) } \\
& =\left\{\langle m, n\rangle / \llbracket v ; g_{\Gamma} \rrbracket(m)=n\right\} .
\end{aligned}
$$

(iii) If $v=\langle 5, w\rangle$, let $\tau_{2}-\tau_{5}$ be primitive recursive functions such that for any sets $A$ and $B$, and any $p, q$,

$$
\begin{gathered}
\left\{\tau_{2}(p)\right\}^{B} \text { enumerates }\{\langle 0,\langle p, n\rangle\rangle \mid\langle p, n\rangle \in B\} ; \\
\left\{\tau_{3}(p)\right\}^{B}(q) \cong\left\{\tau_{2}(p)\right\}^{B}(\alpha(q)) ; \\
\left\{\tau_{4}(e)\right\}^{A}(p) \cong\left\langle 1,\{e\}(\text { least } r:\langle p, r\rangle \in A), \tau_{3}(p)\right\rangle ; \\
\left\{\tau_{5}(e)\right\}^{A}(p) \cong\left\{\tau_{4}(e)\right\}^{A}(\alpha(p)) ;
\end{gathered}
$$

and set $\phi(v, e) \cong\left\langle 1,\{e\}(w), \tau_{5}(e)\right\rangle$.

$$
\begin{aligned}
& {[\psi(v)]=\left[\left\langle 1, \psi(w), \tau_{5}\left(e_{0}\right)\right\rangle\right]=\Gamma\left(\lambda p\left[\left\{\tau_{5}\left(e_{0}\right)\right\}^{\{w(w)]}(p)\right]\right)} \\
& =U\left(\lambda p\left[\left\langle 1, \psi(\text { least } r:\langle p, r\rangle \in[\psi(w)]), \tau_{3}(p)\right\rangle\right]\right) \\
& =U\left(\lambda p \Gamma\left(\lambda q\left[\left\{\tau_{3}(p)\right\}^{\left[\psi \left(\left[w_{i} g_{\Gamma} \prod^{(p))]}\right.\right.\right.}(q)\right]\right)\right) \\
& =U\left(\lambda \dot { p } \cup \left\{[\langle 0,\langle p, n\rangle\rangle] /\left[\llbracket\left[w ; g_{\Gamma} \rrbracket(p) \rrbracket(p)=n\right\}\right)\right.\right. \\
& =\left\{\langle p, n\rangle / \llbracket v ; g_{\Gamma} \rrbracket(p)=n\right\} \text {. }
\end{aligned}
$$

(iv) If $v=\langle 6, w\rangle$, choose $u_{0}$ and $u_{1} \in I^{\Gamma}$ such that $\left[u_{0}\right]=N$ and $\left[u_{1}\right]=\varnothing$, and let $a, b$, and $c$ be indices such that for any $A, B, p$, and $m$,

$$
\begin{aligned}
& \{a\}^{A}(p)=\left(u_{0}, \text { if }\langle p, 0\rangle \in A ; u_{1}, \text { otherwise }\right) ; \\
& \{b\}^{B}(m)=(\langle 0,\langle m, 0\rangle\rangle, \text { if } 0 \in B ;\langle 0,\langle m, 1\rangle\rangle, \text { otherwise }) ; \\
& \{c\}^{B}(m)=\{b\}^{B}(\alpha(m)) .
\end{aligned}
$$

Set $\phi(v, e) \cong\langle 1,\langle 1,\{e\}(w), a\rangle, c\rangle$.

$$
\begin{aligned}
& {[\langle 1, \psi(w), a\rangle]=\Gamma\left(\lambda p\left[\{a\}^{[\psi(w)]}(p)\right]\right)=\Gamma\left(G^{\left[w ; g_{\Gamma} \rrbracket\right.}\right) } \\
= & \left(N, \text { if } g_{\Gamma}\left(\llbracket w ; g_{\Gamma} \rrbracket\right)=0 ; \varnothing \text { otherwise }\right) . \\
{[\psi(v)]=} & \Gamma\left(\lambda m\left[\{c\}^{[\langle 1, \psi(w), a\rangle]}(m)\right]\right) \\
= & \left(\{\langle m, 0\rangle / m \in N\}, \text { if } g_{\Gamma}\left(\llbracket w ; g_{\Gamma} \rrbracket\right)=0 ;\{\langle m, 1\rangle / m \in N\}, \text { otherwise }\right) \\
= & \left\langle\left\langle m, g_{\Gamma}\left(\llbracket w ; g_{\Gamma} \rrbracket\right)\right\rangle / m \in N\right\}=\left\{\langle m, n\rangle / \llbracket v ; g_{\Gamma} \rrbracket(m)=n\right\} .
\end{aligned}
$$

3. Comparisons of effective hierarchies. We have given one criterion for comparison of two operations: if $\Lambda$ subsumes $\Gamma$, then certainly $\mathscr{C}^{\Gamma} \subseteq \mathscr{C}^{\Lambda}$, and intuitively, $\Lambda$ is at least as powerful as $\Gamma$. In this section we establish sufficient conditions to ensure a stronger relationship: $\mathscr{C}^{\Gamma} \subseteq \Delta_{1}^{\Lambda}$.

Definition 3.1. For any analytic operations $\Gamma$ and $\Lambda$ :

(a) $\Lambda$ absorbs $\Gamma$ if and only if there exists a primitive recursive function $\beta$ such that for any family $F$,

$$
\Gamma(\lambda p \Lambda(\lambda q F(\langle p, q\rangle)))=\Lambda(\lambda r F(\beta(r))) ;
$$

(b) $\Lambda$ is normal if and only if it absorbs itself. 
EXAmples. $U$ and $\cap$ are normal with $\beta$ any function which enumerates pairs. $\mathscr{A}$ absorbs $U$ via the function $\beta=\lambda p\left(\left\langle(p)_{0},(p)^{0}\right\rangle\right)$, where $\left(\left\langle m_{0}, m_{1}, \ldots, m_{n}\right\rangle\right)^{0}$ $=\left\langle m_{1}, \ldots, m_{n}\right\rangle . \mathscr{A}$ also absorbs $\cap$ and is normal.

LEMMA 3.2. For any analytic operations $\Gamma, \Gamma_{0}, \Lambda$, and $\Lambda_{0}$ :

(a) if $\Lambda$ absorbs $\Gamma$ and $\Gamma$ subsumes $\Gamma_{0}$, then $\Lambda$ absorbs $\Gamma_{0}$;

(b) if $\Lambda$ absorbs $\Gamma, \Lambda_{0}$ subsumes $\Lambda$, and $\Lambda$ subsumes $\Lambda_{0}$, then $\Lambda_{0}$ absorbs $\Gamma$.

Proof. Let $\Lambda$ absorb $\Gamma$ via $\beta, \Gamma$ subsume $\Gamma_{0}$ via $\alpha, \Lambda_{0}$ subsume $\Lambda$ via $\gamma$, and $\Lambda$ subsume $\Lambda_{0}$ via $\delta$. Let $F$ be any family.

(a) Let $G=\lambda r F\left(\left\langle\alpha\left((r)_{0}\right),(r)_{1}\right\rangle\right)$. Then

$$
\begin{aligned}
\Gamma_{0}(\lambda p \Lambda(\lambda q F(\langle p, q\rangle))) & =\Gamma(\lambda p \Lambda(\lambda q F(\langle\alpha(p), q\rangle))) \\
& =\Gamma(\lambda p \Lambda(\lambda q G(\langle p, q\rangle)))=\Lambda(\lambda r G(\beta(r))) \\
& =\Lambda\left(\lambda r F\left(\left\langle\alpha\left((\beta(r))_{0}\right),(\beta(r))_{1}\right\rangle\right)\right) .
\end{aligned}
$$

(b) Let $H=\lambda r F\left(\left\langle(r)_{0}, \delta\left((r)_{1}\right)\right\rangle\right)$. Then

$$
\begin{aligned}
\Gamma\left(\lambda p \Lambda_{0}(\lambda q F(\langle p, q\rangle))\right) & =\Gamma(\lambda p \Lambda(\lambda q F(\langle p, \delta(q)\rangle))) \\
& =\Gamma(\lambda p \Lambda(\lambda q H(\langle p, q\rangle))) \\
& =\Lambda(\lambda r H(\beta(r)))=\Lambda_{0}(\lambda r H(\gamma(\beta(r)))) \\
& =\Lambda_{0}\left(\lambda r F\left(\left\langle(\gamma(\beta(r)))_{0}, \delta\left((\gamma(\beta(r)))_{1}\right)\right\rangle\right)\right) .
\end{aligned}
$$

LeMma 3.2. For any analytic operation $\Lambda$, any family $F$ over $N$, and any $\alpha$,

$$
\alpha^{-1}(\Lambda(F))=\Lambda\left(\lambda p\left(\alpha^{-1}(F(p))\right)\right)
$$

Proof. Let $G=\lambda p\left(\alpha^{-1}(F(p))\right)$. Then for any $m, p, F^{\alpha(m)}(p)=N \leftrightarrow \alpha(m) \in F(p)$ $\leftrightarrow m \in G(p) \leftrightarrow G^{m}(p)=N$, hence $\alpha(m) \in \Lambda(F) \leftrightarrow \Lambda\left(F^{\alpha(m)}\right)=N \leftrightarrow \Lambda\left(G^{m}\right)=N \leftrightarrow m$ $\in \Lambda(G)$.

The following technical lemma establishes some closure properties of $\Sigma_{1}^{\Lambda}$. The hypothesis on $\Lambda$ is stronger than necessary for some parts of the lemma, but our applications in the next section do not require the stronger results. If $u=\langle 1, v, a\rangle \in I_{1}^{\Lambda}$, we call $u$ a $\Sigma_{1}^{\Lambda}$-index (for $\left.[u ; \Lambda]\right)$.

LEMmA 3.4. For any analytic operations $\Gamma$ and $\Lambda$ such that $\Lambda$ is normal and subsumes $\Gamma, \cup$, and $\cap$ :

(a) $\Sigma_{1}^{\Lambda}$ contains all recursively enumerable sets; precisely, there exists a partial recursive function $\theta_{1}$ such that for any e, $\theta_{1}(e)$ is a $\Sigma_{1}^{\Lambda}$-index for $\{m /\{e\}(m) \cong 0\}$;

(b) $\Sigma_{1}^{\Lambda}$ is closed under recursive counter-image; precisely, there exists a partial recursive function $\theta_{2}$ such that for any $\{e\} \in N^{N}$ and any $\Sigma_{1}^{\Lambda}$-index $u, \theta_{2}(e, u)$ is a $\Sigma_{1}^{\Lambda}$-index for $\{e\}^{-1}([u ; \Lambda])$;

(c) $\Sigma_{1}^{\Lambda}$ is closed under application of $\Gamma$ to recursively enumerated families; precisely, there exists a partial recursive function $\theta_{3}$ such that for any $e$, if $\{e\}(p)$ is a $\Sigma_{1}^{\Lambda}$-index for all $p$, then $\theta_{3}(e)$ is a $\Sigma_{1}^{\Lambda}$-index for $\Gamma(\lambda p[\{e\}(p) ; \Lambda])$;

(d) $\Sigma_{1}^{\Lambda}$ is closed under finite union and intersection; precisely, there exist partial recursive functions $\theta_{4}$ and $\theta_{5}$ such that for any $\Sigma_{1}^{\Lambda}$-indices $u$ and $v, \theta_{4}(u, v)$ is a $\Sigma_{1}^{\Lambda}$ index for $[u ; \Lambda] \cup[v ; \Lambda]$, and $\theta_{5}(u, v)$ is a $\Sigma_{1}^{\Lambda}$-index for $[u ; \Lambda] \cap[v ; \Lambda]$; 
(e) $\Sigma_{1}^{\Lambda}$ is closed under " $\Gamma$-projection"; precisely, there exists a partial recursive function $\theta_{6}$ such that for any $\Sigma_{1}^{\Lambda}$-index $u, \theta_{6}(u)$ is a $\Sigma_{1}^{\Lambda}$-index for

$$
\Gamma(\lambda n\{m /\langle m, n\rangle \in[u ; \Lambda]\}) \text {. }
$$

Proof. (a) follows by the method of definition of $\tau$ in the proof of 2.10. For (b), let $u=\langle 1, v, a\rangle$. Since $u \in I_{1}^{\Lambda}, v \in I_{0}^{\Lambda}$ so we may assume $\{a\}^{[v: \Lambda]}=\{a\}$. Using successively 3.3, (a), and the normality of $\Lambda$, indices $b$ and $c$ may be computed such that

$$
\begin{aligned}
\{e\}^{-1}([u ; \Lambda]) & =\Lambda\left(\lambda p\{e\}^{-1}([\{a\}(p) ; \Lambda])\right) \\
& =\Lambda(\lambda p \Lambda(\lambda q[\{b\}(p, q) ; \Lambda])) \\
& =\Lambda(\lambda r[\{c\}(r) ; \Lambda])=[\langle 1,\langle 0,0\rangle, c\rangle ; \Lambda] .
\end{aligned}
$$

Thus it suffices to take $\theta_{2}(e, u)=\langle 1,\langle 0,0\rangle, c\rangle$. For (c), let $\{e\}(p)=\left\langle 1, v_{p}, a_{p}\right\rangle$. By 3.2 and the hypothesis, $\Lambda$ absorbs $\Gamma$. Hence indices $b$ and $c$ may be computed such that

$$
\begin{aligned}
\Gamma(\lambda p[\{e\}(p) ; \Lambda]) & =\Gamma\left(\lambda p \Lambda\left(\lambda q\left[\left\{a_{p}\right\}(q) ; \Lambda\right]\right)\right) \\
& =\Gamma(\lambda p \Lambda(\lambda q[\{b\}(p, q) ; \Lambda]))=\Lambda(\lambda r[\{c\}(r) ; \Lambda]) .
\end{aligned}
$$

Hence again we may take $\theta_{3}(e, u)=\langle 1,\langle 0,0\rangle, c\rangle$. Part (d) follows easily from (c) with $\Gamma$ replaced by $U$ and $\cap$. For (e) choose $e$ such that for all $m$ and $n$,

Then

$$
\{\{e\}(n)\}(m)=\langle m, n\rangle .
$$

$$
\{m /\langle m, n\rangle \in[u ; \Lambda]\}=\left[\theta_{2}(u,\{e\}(n)) ; \Lambda\right]
$$

and $\theta_{6}$ is easily defined from this and $\theta_{3}$.

THEOREM 3.5. For any analytic operation $\Lambda$ such that $\Lambda$ is normal and subsumes $\cup$ and $\cap, \Delta_{1}^{\Lambda}$ is closed under "recursive in"; precisely, there exists a partial recursive function $\theta_{7}$ such that for any $A, B \subseteq N$ and any $a, u$, and $v$ such that $u$ and $v$ are $\Sigma_{1}^{\Lambda}$-indices for $A$ and $N \sim A$, respectively, and $\{a\}^{A}$ is the characteristic function of $B$, $\theta_{7}(u, v, a)$ is a $\Sigma_{1}^{\Lambda}$-index for $B$.

Proof. We first observe that the second part implies the first; $N \sim B$ is also recursive in $A$ and another value of $\theta_{7}$ yields a $\Sigma_{1}^{\Lambda}$-index for $N \sim B$. Let $R$ be a recursive relation such that

$$
m \in B \leftrightarrow \exists s\left(R(s, m) \wedge \forall n_{n<\ln (s)}\left[\left(n \in A \wedge(s)_{n}=0\right) \vee\left(n \notin A \wedge(s)_{n}=1\right)\right]\right) .
$$

Using (a) and (d) of the previous lemma, a $\Sigma_{1}^{\Lambda}$-index $w$ may be computed so that

$$
\begin{aligned}
& {[w ; \Lambda]=\left\{\langle\langle m, s\rangle, n\rangle / R(s, m) \wedge\left[(n \geqq \ln (s)) \vee\left(n \in A \wedge(s)_{n}=0\right)\right.\right.} \\
&\left.\left.\vee\left(n \notin A \wedge(s)_{n}=1\right)\right]\right\} .
\end{aligned}
$$

Then if $\theta_{6}^{1}$ and $\theta_{6}^{2}$ are as in 3.4(e) for $\Gamma=U$ and $\Gamma=\cap$, respectively,

$$
\begin{aligned}
B & =\cup(\lambda s\{m /\langle m, s\rangle \in \cap(\lambda n\{p /\langle p, n\rangle \in[w ; \Lambda]\})\}) \\
& =\cup\left(\lambda s\left\{m /\langle m, s\rangle \in\left[\theta_{6}^{2}(w) ; \Lambda\right]\right\}\right)=\left[\theta_{6}^{1}\left(\theta_{6}^{2}(w)\right) ; \Lambda\right] .
\end{aligned}
$$

Lemma 3.6. For any analytic operations $\Gamma$ and $\Lambda$ such that $\Lambda$ is normal and subsumes $\Gamma, U$, and $\cap, \Sigma_{1}^{\Lambda}$ is closed under application of $\Gamma$ to families recursively enumerated relative to $\Delta_{1}^{\Lambda}$ sets; precisely, there exists a partial recursive function $\theta_{8}$ 
such that for any $A \subseteq N$, any $u, v$ which are $\Sigma_{1}^{\Lambda}$-indices for $A$ and $N \sim A$, respectively, and any $e$ such that for all $p,\{e\}^{A}(p)$ is a $\Sigma_{1}^{\Lambda}$-index, $\theta_{8}(e, u, v)$ is a $\Sigma_{1}^{\Lambda}$-index for $\Gamma\left(\lambda p\left[\{e\}^{A}(p) ; \Lambda\right]\right)$.

\section{Proof. Let}

$$
B=\left\{\langle m, p, n, q\rangle \mid \exists w a\left(\{e\}^{A}(p)=\langle 1, w, a\rangle \wedge\{a\}^{[w ; \Lambda]}(q)=\langle 0, m\rangle\right)\right\} .
$$

For $e$ and $A$ which satisfy the hypothesis, $w$ will be in $I_{0}^{\Lambda}$ and thus $B$ is easily seen to be recursive in $A$. Hence, by the previous theorem, from $u$ and $v$ we can compute a $\Sigma_{1}^{\Lambda}$-index for $B$. The desired set is obtained from $B$ by successive "projections" under $\Gamma, U$, and $\Gamma$, and thus a $\Sigma_{1}^{\Lambda}$-index for it may be computed by 3.4(e).

Since $\mathscr{C}^{\Gamma}$ is closed under complementation, $\mathscr{C}^{\Gamma} \subseteq \Delta_{1}^{\Lambda}$ follows from $\mathscr{C}^{\Gamma} \subseteq \Sigma_{1}^{\Lambda}$. Of course, in general $\Sigma_{1}^{\Lambda}$ is not closed under complementation so a straightforward induction over $I^{\Gamma}$ to establish the latter inclusion would seem to be blocked. Use of the operation $\Gamma^{\circ}$ dual to $\Gamma$, enables us to avoid complementation in generating $\mathscr{C} \Gamma$.

Definition 3.7. For any operation $\Gamma$ and any $F \in \mathscr{P}(N)^{N}$ :

$$
\Gamma^{\circ}(F)=N \sim \Gamma(\lambda p(N \sim F(p))) .
$$

It is easy to check that if $\Gamma$ is analytic, so is $\Gamma^{\circ}$, and that for any $\langle 1, u, a\rangle \in I^{\Gamma}$,

$$
[\langle 2,\langle 1, u, a\rangle\rangle ; \Gamma]=\Gamma^{\circ}\left(\lambda p\left[\left\langle 2,\{a\}^{[u ; \Gamma]}(p)\right\rangle ; \Gamma\right]\right) .
$$

EXAMPLES. $U^{\circ}=n,(\lim \text { inf })^{\circ}=\lim$ sup, and

$$
\mathscr{A}^{\circ}(F)=\{m / \forall \beta \exists x(m \in F(\bar{\beta}(x)))\} .
$$

THEOREM 3.8. For any analytic operations $\Gamma$ and $\Lambda$ such that $\Lambda$ is normal and subsumes $\Gamma, \Gamma^{\circ}, \cup$, and $\cap$ :

$$
\mathscr{C}^{\Gamma} \subseteq \Delta_{1}^{\Lambda}
$$

In fact, there exists a partial recursive function $\psi$ such that for all $u \in I^{\Gamma}, \psi(u)$ is a $\Sigma_{1}^{\Lambda}$-index for $[u ; \Gamma]$.

Proof. We define $\psi$ via the recursion theorem as $\left\{e_{0}\right\}$, where $\left\{e_{0}\right\}(u) \cong \phi\left(u, e_{0}\right)$ and $\phi$ is defined by cases as follows.

(i) If $u=\langle 0, m\rangle$ or $\langle 2,\langle 0, m\rangle\rangle, \phi(u, e)$ is defined using 3.4(a).

(ii) If $u=\langle 1, v, a\rangle$, let $\sigma_{1}$ and $\sigma_{2}$ be primitive recursive functions such that

$$
\begin{aligned}
\left\{\sigma_{1}(u, e)\right\}^{A}(p) & \cong\{e\}\left(\{a\}^{A}(p)\right), \\
\sigma_{2}(u, e) & =\left((v)_{1}, \text { if }(v)_{0}=2 ;\langle 2, v\rangle, \text { otherwise }\right),
\end{aligned}
$$

and set $\phi(u, e) \cong \theta_{8}\left(\sigma_{1}(u, e),\{e\}(v),\{e\}\left(\sigma_{2}(u, e)\right)\right)$.

$$
\begin{aligned}
{[\psi(u) ; \Lambda] } & =\left[\phi\left(u, e_{0}\right) ; \Lambda\right]=\Gamma\left(\lambda p\left[\left\{\sigma_{1}\left(u, e_{0}\right)\right\}^{[\psi(v) ; \Lambda]}(p) ; \Lambda\right]\right) \\
& =\Gamma\left(\lambda p\left[\psi\left(\{a\}^{[\psi(v) ; \Lambda]}(p) ; \Lambda\right)\right]\right) \\
& =\Gamma\left(\lambda p\left[\{a\}^{[v: \Gamma]}(p) ; \Gamma\right]\right) \quad \text { (Induction hypothesis) } \\
& =[u ; \Gamma] .
\end{aligned}
$$


(iii) If $u=\langle 2,\langle 1, v, a\rangle\rangle$, we use the remark following 3.7 and proceed as in (ii).

(iv) If $u=\langle 2,\langle 2, v\rangle\rangle, \phi(u, e) \cong \phi(v, e)$.

4. The $R$-operator. Although the discovery of the operator $R$ is generally ascribed to Kolmogorov (cf. [Ka-Li, II, p. 82]), he seems never to have published any account of it. Much of its elementary theory was developed first in [Ka-Li, II], and further results obtained in $[\mathbf{L j} \mathbf{1}, \mathbf{2}]$. Much of the material of this section is adapted from these sources. Definition 4.2 is due to Hausdorff [Ha, p. 103].

Definition 4.1. For any analytic operation $\Gamma$ :

(a) $\Gamma$ is isotone if and only if for any families $F$ and $G$ such that $\forall p(F(p) \subseteq G(p))$, also $\Gamma(F) \subseteq \Gamma(G)$;

(b) $\Gamma$ is positive if and only if $\Gamma$ is isotone and nontrivial-that is, for some families $F$ and $G, \Gamma(F) \neq \Gamma(G)$.

Definition 4.2. For any $M \subseteq N^{N}, \Lambda_{M}$ is the operation defined by:

$$
\Lambda_{M}(F)=\left\{m / \exists \beta_{\beta \in M} \forall x(m \in F(\beta(x)))\right\} .
$$

$M$ is called a base of $\Lambda_{M}$.

EXAMPLES. If $M=\{\lambda x(p) \mid p \in N\}$, then $\Lambda_{M}=U$. If $M=\left\{\bar{\beta} \mid \beta \in N^{N}\right\}, \Lambda_{M}=\mathscr{A}$.

Note that if $M \subseteq M^{\prime}$ and for every $\gamma \in M^{\prime}$ there is a $\beta \in M$ such that $\forall x \exists y(\beta(x)$ $=\gamma(y))$, then $\Lambda_{M}=\Lambda_{M^{\prime}}$. The largest set $M^{\prime}$ for which this is true is called the canonical base for $\Lambda_{M}$.

LEMMA 4.3. For any operation $\Gamma, \Gamma$ is positive analytic if and only if for some nonempty $M \subseteq N^{N}, \Gamma=\Lambda_{M}$.

Proof. It is obvious that every $\Lambda_{M}$ is analytic and isotone. If $M \neq \varnothing, \Lambda_{M}(\lambda p(\varnothing))$ $=\varnothing$, while $\Lambda_{M}(\lambda p(N))=N$, so $\Lambda_{M}$ is nontrivial, hence positive analytic. Conversely, suppose $\Gamma$ is positive analytic and let

$$
M=\left\{\beta / \forall F \in \mathscr{P}(N)^{N}[\cap(\lambda p F(\beta(p))) \subseteq \Gamma(F)]\right\} .
$$

Then $\Gamma=\Lambda_{M}$.

Definition 4.4. For any $P \in \mathscr{P}\left(N^{N}\right)^{N}$ and any $S \subseteq N$ :

(a) $S$ is a $P$-fan if and only if

(i) $\varnothing \in S$,

(ii) $\forall s t(s \subseteq t \in S \rightarrow s \in S)$, and

(iii) $\forall s_{s \in S} \exists \beta_{\beta \in P(s)} \forall x(s\langle\beta(x)\rangle \in S)$;

(b) $R P=\{\gamma /$ range $(\gamma)$ is a $P$-fan $\}$.

DEFINITION 4.5. For any family $\lambda s \Gamma_{s}$ of positive analytic operations, let, for each $s, P(s)$ be the canonical base for $\Gamma_{s}$. Then $R\left(\lambda s \Gamma_{s}\right)=\Lambda_{R P}$.

In many of our applications of the last two definitions, $\forall s\left(\Gamma_{s}=\Gamma=\Lambda_{M}\right)$, and in this case we write simply $R \Gamma=\Lambda_{R M}$, where $R M=R(\lambda s M)$. We defined $R\left(\lambda s \Gamma_{s}\right)$ via the canonical bases for $\Gamma_{s}$ to ensure independence of choice of base, but it is easy to check that if for each $s, Q(s)$ is another base for $\Gamma_{s}$, then $R\left(\lambda s \Gamma_{s}\right)=\Lambda_{R Q}$.

EXAMPLES. For any $m$ and $F, m \in R \Gamma(F)$ if and only if $\{p / m \in F(p)\}$ includes some $M$-fan, where $\Gamma=\Lambda_{M}$. If $M=\{\lambda x(p) / p \in N\}$, an $M$-fan is any set of the form 
$\{\beta(x) / x \in N\}$. Thus $R \cup=\Lambda_{R M}=\mathscr{A}$. If $M=\{\lambda x(x)\}, N$ itself is the only $M$-fan, so $R \cap=\cap$.

LEMMA 4.6. For any positive analytic operations $\Gamma$ and $\Gamma_{1}$ :

(a) $R \Gamma$ subsumes $\Gamma$;

(b) $R \Gamma$ absorbs $\Gamma$;

(c) if $\Gamma$ subsumes $\Gamma_{1}$, then $R \Gamma$ subsumes $R \Gamma_{1}$.

Proof. Let $M$ be the canonical base for $\Gamma$.

(a) We claim $\Gamma(F)=R \Gamma\left(\lambda p F\left((p)_{0}\right)\right)$, for any $F$. Suppose first $m$ is in the right-hand side. Then for some $M$-fan $S, \forall s_{s \in S}\left(m \in F\left((s)_{0}\right)\right)$. Since $\varnothing \in S$, for some $\beta \in M$, $\forall x(\langle\beta(x)\rangle \in S)$ and thus $\forall x(m \in F(\beta(x)))$. Hence $m \in \Gamma(F)$. The converse inclusion is obvious.

(b) Let $\left(\left\langle m_{0}, m_{1}, \ldots, m_{n}\right\rangle\right)^{0}=\left\langle m_{1}, \ldots, m_{n}\right\rangle$ for any $m_{i}$. Then it is easy to check that for any $F$,

$$
\Gamma(\lambda p R \Gamma(\lambda q F(\langle p, q\rangle)))=R \Gamma\left(\lambda r F\left(\left\langle(r)_{0},(r)^{0}\right\rangle\right)\right) .
$$

(c) Let $\alpha$ be a primitive recursive function by which $\Gamma$ subsumes $\Gamma_{1}$ and define $\beta$ by

$$
\beta\left(\left\langle m_{0}, \ldots, m_{n}\right\rangle\right)=\left\langle\alpha\left(m_{0}\right), \ldots, \alpha\left(m_{n}\right)\right\rangle .
$$

Then $R \Gamma_{1}(F)=R \Gamma(\lambda p(\beta(p)))$.

The second example above demonstrates that $R \Gamma$ may in general be no more powerful than $\Gamma$ itself. In particular, to apply Theorem 3.8 we need an operation $\Lambda$ which subsumes both $\Gamma$ and $\Gamma^{\circ}$, and is normal. The first requirement is satisfied by

$$
\Gamma^{*}(F)=\Gamma\left(\lambda p \Gamma^{\circ}(\lambda q F(\langle p, q\rangle))\right),
$$

since $\Gamma(F)=\Gamma^{*}\left(\lambda r F\left((r)_{0}\right)\right)$ and $\Gamma^{\circ}(F)=\Gamma^{*}\left(\lambda r F\left((r)_{1}\right)\right)$. Normality is achieved by applying $R$ :

LEMMA 4.7. For any positive analytic operation $\Gamma, R \Gamma$ is normal.

We defer briefly the proof. Let $R^{*} \Gamma=R \Gamma^{*}$.

THEOREM 4.8. For any positive analytic operation $\Gamma$ which subsumes U:

$$
\mathscr{C}^{\Gamma} \subseteq \Delta_{1}^{R^{*} \Gamma}
$$

Proof. If $\Gamma$ subsumes $U$, then $\Gamma^{\circ}$ subsumes $U^{\circ}=n$, hence all four are subsumed by $R^{*} \Gamma$. The theorem follows by 3.8 and 4.7 .

The proof of 4.7 is a mass of combinatorial detail and we recommend that all but the stoutest of heart take it on faith. Since by $4.6, R R \Gamma$ absorbs $R \Gamma$, it suffices by 3.2 to show that $R \Gamma$ subsumes $R R \Gamma$. For any $s$, let $t=\ln (s)-1$ and for $a<\ln (t)$ and $b \leqq(t)_{a}$, let

$$
\begin{aligned}
\phi(s, a, b) & =(s)_{(t \mid a)\langle b\rangle}, \\
\psi(s, a) & =\left\langle\phi(s, a, 1), \ldots, \phi\left(s, a,(t)_{a}\right)\right\rangle \\
\theta(s) & =\langle\psi(s, 0), \psi(s, 1), \ldots, \psi(s, \ln (t)-1)\rangle .
\end{aligned}
$$


Then we shall show that for any family $F$,

$$
R R \Gamma(F)=R \Gamma(\lambda p F(\theta(p))) .
$$

Let $M$ be the canonical base for $\Gamma$. Then $R M$ is a base for $R \Gamma$ and $R R M$ for $R R \Gamma$. It will suffice to show:

(a) for any $M$-fan $S, \theta^{*}(S)=\{\theta(s) / s \in S\}$ is an $R M$-fan;

(b) for any $R M$-fan $T, \theta^{-1}(T)$ includes an $M$-fan.

A few auxiliary formulas will make the proof easier. Let $s$ and $s_{0}$ be arbitrary, $t=\ln (s)-1$, and $t_{0}=\ln \left(s_{0}\right)-1$. We show first:

(c) $\ln (\theta(s))=\ln (t)$;

(d) $\theta\left(s_{0}\right) \subseteq \theta(s) \rightarrow t_{0} \subseteq t$

(e) $s_{0} \subseteq s \wedge t_{0} \subseteq t \rightarrow \theta\left(s_{0}\right)=\theta(s) \mid \ln \left(t_{0}\right)$;

(f) $\theta(s)=\theta\left(s_{0}\right)\langle w\rangle \rightarrow t=t_{0}\langle\ln (w)\rangle$;

(g) $s_{0} \subseteq s \wedge t=t_{0}\langle m\rangle \rightarrow \theta(s)=\theta\left(s_{0}\right)\langle w\rangle$ for some $w$ such that $\ln (w)=m$.

Furthermore, for any $r\left\langle m\right.$, if $t_{2}=t\langle r\rangle$ and $\left.s_{2}=s\right|\left(t_{2}+1\right)$, then $\theta\left(s_{2}\right)=\theta\left(s_{0}\right)\langle w \mid r\rangle$.

Formula (c) is obvious by inspection. Let $p=\ln (t)-1$ and $p_{0}=\ln \left(t_{0}\right)-1$; then

$$
\theta(s)=\langle\psi(s, 0), \psi(s, 1), \ldots, \psi(s, p)\rangle
$$

and

$$
\theta\left(s_{0}\right)=\left\langle\psi\left(s_{0}, 0\right), \psi\left(s_{0}, 1\right), \ldots, \psi\left(s_{0}, p_{0}\right)\right\rangle .
$$

For (d), suppose $\theta(s) \subseteq \theta\left(s_{0}\right)$. Hence $p_{0} \leqq p$ and for $q \leqq p_{0}, \psi(s, q)=\psi\left(s_{0}, q\right)$. Hence $\left(t_{0}\right)_{q}=\ln \left(\psi\left(s_{0}, q\right)\right)=\ln (\psi(s, q))=(t)_{q}$ and $t_{0} \subseteq t$. For (e), suppose $s_{0} \subseteq s$ and $t_{0} \subseteq t$. Then $s_{0}=s \mid\left(t_{0}+1\right)$. For $a \leqq p_{0}$ and $b<\left(t_{0}\right)_{a},\left(t_{0} \mid a\right)\langle b\rangle\left\langle t_{0}\right.$, so $\phi\left(s_{0}, a, b\right)=\left(s_{0}\right)_{\left(t_{0} \mid a\right)\langle b\rangle}$ $=(s)_{\left(t_{0} \mid a\right)\langle b\rangle}=(s)_{(t \mid a)\langle b\rangle}=\phi(s, a, b)$. Since also $\left(t_{0}\right)_{a}=(t)_{a}, \psi\left(s_{0}, a\right)=\psi(s, a)$, and $\theta\left(s_{0}\right)=\theta(s) \mid \ln \left(t_{0}\right)$ follows immediately. For (f), if $\theta(s)=\theta\left(s_{0}\right)\langle w\rangle$, it follows from (c) and (d) that $t=t_{0}\langle m\rangle$ for some $m$. But

$$
w=\psi(s, p)=\left\langle\phi(s, p, 1), \ldots, \phi\left(s, p,(t)_{p}\right)\right\rangle
$$

and $\ln (w)=(t)_{p}=m$. For (g), if $s_{0} \subseteq s$ and $t=t_{0}\langle m\rangle$, it follows by (c), (e), and (f) that $\theta(s)=\theta\left(s_{0}\right)\langle w\rangle$ for some $w$ such that $\ln (w)=m$. If $r\left\langle m, t_{2}=t\langle r\rangle\right.$, and $\left.s_{2}=s\right|\left(t_{2}+1\right)$, then for $a<p, \psi(s, a)=\psi\left(s_{2}, a\right)$, and $\psi\left(s_{2}, p\right)=\psi(s, p)\left|\left(t_{2}\right)_{p}=w\right| r$.

To prove (a), let $S$ be any $M$-fan and $T=\theta^{*}(S)$. We must show that $T$ is an $R M$-fan. (i) Since $\varnothing \in S$ and $\theta(\varnothing)=\varnothing, \varnothing \in T$. (ii) Suppose $u=\theta(s) \in T$ and $v \subseteq u$. Then setting $t=\ln (s)-1, t_{0}=t \mid \ln (v)$, and $s_{0}=s \mid\left(t_{0}+1\right)$, it follows from (e) that $\theta\left(s_{0}\right)=v$. Since $s_{0} \subseteq s \in S, s_{0} \in S$ and thus $v \in T$. (iii) Suppose $u=\theta(s) \in T$ and $W=\{w / u\langle w\rangle \in T\}$. We must show that $W$ is an $M$-fan. (1) Since $S$ is an $M$-fan, there exists an $s_{1} \in S$ such that $s \subseteq s_{1}$ and if $t=\ln (s)-1$ and $t_{1}=t\langle 0\rangle$, then $t_{1}$ $=\ln \left(s_{1}\right)-1$. Then by $(\mathrm{g}), \theta\left(s_{1}\right)=\theta(s)\langle\varnothing\rangle=u\langle\varnothing\rangle \in T$. Hence $\varnothing \in W$. (2) Suppose $v \subseteq w \in W$; then for some $s_{1} \in S, \theta\left(s_{1}\right)=u\langle w\rangle$. Let $t_{1}=\ln \left(s_{1}\right)-1$. By (f), $t_{1}=t\langle\ln (w)\rangle$. Let $r=\ln (v), t_{2}=t\langle r\rangle$, and $s_{2}=s_{1} \mid\left(t_{2}+1\right)$. By $(\mathrm{g}), \theta\left(s_{2}\right)=u\langle w \mid r\rangle=u\langle v\rangle$. Since $s_{2} \subseteq s_{1} \in S, u\langle v\rangle \in T$ and $v \in W$. (3) Suppose $w \in W, \theta\left(s_{1}\right)=u\langle w\rangle$, and $t_{1}=\ln \left(s_{1}\right)-1$ 
$=t\langle\ln (w)\rangle$. Let $s_{3}$ be a member of $S$ such that $s_{1} \subseteq s_{3}$ and $\ln \left(s_{3}\right)=t\langle\ln (w)+1\rangle$. Then by (g), for any $m$, if $p=\ln (t)$,

$$
\theta\left(s_{3}\langle m\rangle\right)=u\left\langle w\left\langle\phi\left(s_{3}\langle m\rangle, p, \ln (w)+1\right)\right\rangle\right\rangle=u\langle w\langle m\rangle\rangle .
$$

Since $S$ is an $M$-fan, there exists a $\beta \in M$ such that $\forall x\left(s_{3}\langle\beta(x)\rangle \in S\right)$. Hence, $\forall x(u\langle w\langle\beta(x)\rangle\rangle \in T)$ and $\forall x(w\langle\beta(x)\rangle \in W)$.

To prove (b), let $T$ be any $R M$-fan and

$$
S=\left\{s / \forall u_{u \subseteq s}(\theta(u) \in T)\right\} .
$$

Then $S \subseteq \theta^{-1}(T)$ and it suffices to show that $S$ is an $M$-fan. Trivially, $\varnothing \in S$ and $s \subseteq t \in S \rightarrow s \in S$. Suppose $s \in S$ and $t=\ln (s)$. Assuming $t \neq \varnothing$ (a slight modification is required in this case), there exists $t_{0}$ and $m$ such that $t=t_{0}\langle m\rangle$. Let $s_{0}=s \mid\left(t_{0}+1\right)$. Then by (g), for every $n$ there exists a $w_{n}$ such that $\theta(s\langle n\rangle)=\theta\left(s_{0}\right)\left\langle w_{n}\right\rangle$. If $m=0$, $w_{n}=\varnothing$ for all $n$ so $\forall n(\theta(s\langle n\rangle) \in T)$ and any $\beta \in M$ is such that $\forall x(s\langle\beta(x)\rangle \in T)$. If $m>0$ there exists a $v$ such that for all $n$

$$
w_{n}=v\langle\phi(s\langle n\rangle, \ln (t)-1, m)\rangle=v\langle n\rangle .
$$

By the second part of (g), if $t_{2}=t_{0}\langle m-1\rangle$, and $s_{2}=s \mid\left(t_{2}+1\right)$, then $\theta\left(s_{2}\right)=\theta\left(s_{0}\right)\langle v\rangle$. Since $s_{2} \subseteq s \in S, \theta\left(s_{2}\right) \in T$. Since $T$ is an $R M$-fan, $\left\{u \mid \theta\left(s_{0}\right)\langle u\rangle \in T\right\}$ is an $M$-fan. Thus there exists $\beta \in M$ such that $\forall x\left(\theta\left(s_{0}\right)\langle v\langle\beta(x)\rangle\rangle \in T\right)$. Hence, $\forall x(\theta(s\langle\beta(x)\rangle) \in T)$ and $\forall x(s\langle\beta(x)\rangle \in S)$.

5. Extended functionals. The remainder of this paper is devoted to showing that the inclusion of Theorem 4.8 may be proper and obtaining concurrently a recursion-theoretic characterization of $\Delta_{1}^{R^{*} \Gamma}$.

According to Kleene's schema $S 8$ (or $S 0$ ) [KI 3, 3.7], a computation of the form $f(\lambda x \phi(f, x))$ is defined just in case $\operatorname{Dm}(\lambda x \phi(f, x))=N$. Since $\operatorname{Dm}(f)=N^{N}$, this is equivalent to: $\lambda x \phi(f, x) \in \operatorname{Dm}(f)$. Let $f^{\#}$ be a function with range included in $N$ and domain included in the set of partial functions from $N$ into $N$, and suppose $f^{\#}$ is consistent-i.e., if $\phi \in \mathrm{Dm}\left(f^{\#}\right)$ and $\psi$ extends $\phi$, then $f^{\#}(\phi)=f^{\#}(\psi)$. Then $S 1-S 9$, with $S 8$ altered to require $\lambda x \phi\left(f^{\#}, x\right) \in \operatorname{Dm}\left(f^{\#}\right)$, define a notion of recursive computation relative to $f^{\#}$ which coincides with the usual notion when $\operatorname{Dm}\left(f^{\#}\right)$ $=N^{N}$.

Of course, we cannot expect that all of the standard theorems will go through for this expanded notion. For example, the representation theorems [KI 3, XXVI and XXVIII] almost certainly will not hold in the same form. On the other hand many of the simpler results such as the recursion theorem and the substitution theorem [KI 3, XXII] are not affected by this alteration and continue to hold.

Definition 5.1. For any positive analytic operation $\Gamma$ with canonical base $M$ and any partial function $\phi: N \rightarrow N$ :

(a) $g_{\Gamma}^{\#}(\phi) \cong 0 \leftrightarrow \exists \beta_{\beta \in M} \forall x(\phi(\beta(x)) \cong 0)$;

(b) $g_{\Gamma}^{\#}(\phi) \cong 1 \leftrightarrow \forall \beta_{\beta \in M} \exists x(\phi(\beta(x)) \cong 1)$;

(c) otherwise, $\phi \notin \operatorname{Dm}\left(g_{\Gamma}^{\#}\right)$. 
Since the condition for $\varphi \in \operatorname{Dm}\left(g_{\Gamma}^{\#}\right)$ is a requirement that "sufficiently many" values of $\varphi$ have a certain value, $g_{\Gamma}^{\#}$ is consistent. The next definition and several of the theorems about it were inspired by Moschovakis' [Mo 2]. Throughout the remainder of this section $\Gamma$ denotes a positive analytic operation with canonical base $M$, and PRI is the set of indices of primitive recursive functions.

DEFINITION 5.2. $K^{\Gamma}$ is the smallest subset of $N$ such that for any $a \in \mathrm{PRI}$ :

(a) $0 \in K^{\Gamma}$;

(b) $\exists \beta_{\beta \in M} \forall y\left[\{a\}(\beta(y)) \in K^{\Gamma}\right] \rightarrow\langle 1, a\rangle \in K^{\Gamma}$;

(c) $\forall \beta_{\beta \in M} \exists y\left[\{a\}(\beta(y)) \in K^{\Gamma}\right] \rightarrow\langle 2, a\rangle \in K^{\Gamma}$.

LEMMA 5.3. If $\Gamma$ subsumes $U$, there exist primitive recursive functions $\pi_{\wedge}, \pi_{\vee}, \pi_{\exists}$, and $\pi_{\forall}$ such that for any $u$ and $v$ and any $a \in \mathrm{PRI}$ :

(a) $u \in K^{\Gamma}$ and $v \in K^{\Gamma} \leftrightarrow \pi_{\wedge}(u, v) \in K^{\Gamma}$;

(b) $u \in K^{\Gamma}$ or $v \in K^{\Gamma} \leftrightarrow \pi_{\mathrm{\vee}}(u, v) \in K^{\Gamma}$;

(c) $\exists x\left[\{a\}(x) \in K^{\Gamma}\right] \leftrightarrow \pi_{\exists}(a) \in K^{\Gamma}$;

(d) $\forall x\left[\{a\}(x) \in K^{\Gamma}\right] \leftrightarrow \pi_{\forall}(a) \in K^{\Gamma}$.

Furthermore, in the natural well-ordering of $K^{\Gamma}$ induced by its inductive definition, if $u, v \in K^{\Gamma}$ then they precede $\pi_{\wedge}(u, v)$, if one of $u, v$ belongs to $K^{\Gamma}$ then it precedes $\pi_{\mathrm{v}}(u, v)$, etc.

Proof. Let $\pi_{1}$ be a primitive recursive function so that $\left\{\pi_{1}(u, v)\right\}(0)=u$ and $\left\{\pi_{1}(u, v)\right\}(m+1)=v$. Then we may take

$$
\pi_{\wedge}(u, v)=\pi_{\forall}\left(\pi_{1}(u, v)\right) \text { and } \pi_{\vee}(u, v)=\pi_{\exists}\left(\pi_{1}(u, v)\right),
$$

so it suffices to establish (c) and (d). Let $\alpha$ be the primitive recursive function by which $\Gamma$ subsumes $U$ and $\pi_{2}$ a primitive recursive function such that $\left\{\pi_{2}(a)\right\}$ $=\lambda x\{a\}(\alpha(x))$ for any $a \in \mathrm{PRI}$. Then

$$
\begin{aligned}
\exists x\left[\{a\}(x) \in K^{\Gamma}\right] & \leftrightarrow \exists \beta_{\beta \in M} \forall x\left[\{a\}(\alpha(\beta(y))) \in K^{\Gamma}\right] \\
& \leftrightarrow\left\langle 1, \pi_{2}(a)\right\rangle \in K^{\Gamma} .
\end{aligned}
$$

Hence $\pi_{\exists}(a)=\left\langle 1, \pi_{2}(a)\right\rangle$ will do. Likewise, $\pi_{\forall}(a)=\left\langle 2, \pi_{2}(a)\right\rangle$.

THEOREM 5.4. If $\Gamma$ subsumes $\cup, K^{\Gamma}$ is complete for computations in $g_{\Gamma}^{\#}$; precisely, there exists a primitive recursive function $\rho$ such that for any $a, s$, and $n$,

$$
\{a\}\left(g_{\Gamma}^{\#}, s\right) \cong n \leftrightarrow \rho(a, s, n) \in K^{\Gamma} .
$$

Proof. We define $\rho$ by the recursion theorem in terms of its own index $r$. It will be clear at the end that $\rho$ so defined is in fact primitive recursive. The proofs that $\rho$ has the desired properties are by straight forward inductions over computations and over $K^{\Gamma}$, respectively, and aside from indications given during the construction of $\rho$, will be left to the reader. The definition is by cases determined by which schema $S(a)_{0}$ applies. Note that $1 \notin K^{\Gamma}$.

$(a)_{0}=1,2$, or $3: \rho(a, s, n)=\left(0\right.$, if $\{a\}\left(g_{\Gamma}^{\#}, s\right)=n ; 1$, otherwise $)$.

$(a)_{0}=4$ : Let $\rho_{1}$ be a primitive recursive function such that

$$
\left\{\rho_{1}(r, a, s, n)\right\} \cong \pi_{\wedge}\left(\{r\}\left((a)_{3}, s, p\right),\{r\}\left((a)_{2}, s\langle p\rangle, n\right)\right) .
$$


Then since

$$
\{a\}\left(g_{\Gamma}^{\#}, s\right) \cong n \leftrightarrow \exists p\left[\left\{(a)_{3}\right\}\left(g_{\Gamma}^{\#}, s\right) \cong p \wedge\left\{(a)_{2}\right\}\left(g_{\Gamma}^{\#}, s\langle p\rangle\right) \cong n\right],
$$

it suffices to set $\rho(a, s, n)=\pi_{\ni}\left(\rho_{1}(r, a, s, n)\right)$.

$(a)_{0}=5:$ In this case

$$
\begin{aligned}
\{a\}\left(g_{\Gamma}^{\#}, s\langle m\rangle\right) & \cong n \leftrightarrow \\
\exists p\left[( m = 0 \wedge \{ ( a ) _ { 2 } \} ( g _ { \Gamma } ^ { \# } , s ) \cong n ) \vee \left(m>0 \wedge\{a\}\left(g_{\Gamma}^{\#}, s\langle m-1\rangle\right) \cong p\right.\right. & \left.\left.\wedge\left\{(a)_{3}\right\}\left(g_{\Gamma}^{\#}, s\langle p\rangle\right) \cong n\right)\right] .
\end{aligned}
$$

Hence the techniques of the previous case lead to the appropriate value for $\rho(a, s, n)$.

$(a)_{0}=6$ : Let $s^{\prime}$ be the prescribed rearrangement of $s$ so that

$$
\{a\}\left(g_{\Gamma}^{\#}, s\right) \cong\left\{(a)_{4}\right\}\left(g_{\Gamma}^{\#}, s^{\prime}\right)
$$

and let $\rho_{2}$ be a primitive recursive function such that

$$
\left\{\rho_{2}(r, a, s, n)\right\}(p) \cong\{r\}\left((a)_{4}, s^{\prime}, n\right) .
$$

Then set $\rho(a, s, n)=\pi_{\exists}\left(\rho_{2}(r, a, s, n)\right)$.

$(a)_{0}=7$ : does not arise.

$(a)_{0}=8:$ Let $\rho_{3}(n, i)=(0$, if $n=i ; 1$, otherwise $)$, and

$$
\left\{\rho_{4}(r, a, s, i)\right\}(p) \cong\{r\}\left((a)_{3}, s\langle p\rangle, i\right) .
$$

Set $\rho(a, s, n)=\pi_{\vee}\left(\pi_{\wedge}\left(\rho_{3}(n, 0),\left\langle 1, \rho_{4}(r, a, s, 0)\right\rangle\right), \pi_{\wedge}\left(\rho_{3}(n, 1),\left\langle 2, \rho_{4}(r, a, s, 1)\right\rangle\right)\right)$.

$(a)_{0}=9$ : Let $t$ be the subsequence of $s$ such that

$$
\{a\}\left(g_{\Gamma}^{\#}, s\right) \cong\left\{(s)_{0}\right\}\left(g_{\Gamma}^{\#}, t\right),
$$

choose $\rho_{5}$ primitive recursive such that

$$
\left\{\rho_{5}(r, a, s, n)\right\}(p) \cong\{r\}\left((s)_{0}, t, n\right),
$$

and set $\rho(a, s, n)=\pi_{\exists}\left(\rho_{5}(r, a, s, n)\right)$. Otherwise: Set $\rho(a, s, n)=1$.

It is clear that any function recursive in $g_{\Gamma}$ is also recursive in $g_{\Gamma}^{\#-i n ~ f a c t, ~ i f ~}$ $\operatorname{sg}(x)=(0$, if $x=0 ; 1$, otherwise $)$, then $g_{\Gamma}(\alpha)=g_{\Gamma}^{\#}(\lambda x \operatorname{sg}(\alpha(x)))$. We state this formally in the following equivalent (by 2.10) form. The proof is by an obvious construction and is omitted.

LEMMA 5.5. There exists a primitive recursive function $\sigma$ such that for all $u$ and $m$, $u \in I^{\Gamma} \rightarrow \lambda m\{\sigma(u)\}\left(g_{\Gamma}^{\#}, m\right)$ is the characteristic function of $[u ; \Gamma]$.

The goal of the rest of this section is to show that if $\Gamma$ subsumes $\mathscr{A}$, then ${ }_{1} \mathrm{sc}\left(g_{\Gamma}^{\#}\right)$ is properly greater than ${ }_{1} \mathrm{sc}\left(g_{\Gamma}\right)$. A simple diagonal argument shows that $I^{\Gamma}$ is not recursive in $g_{\Gamma}$. Our plan is to show that both $I^{\Gamma}$ and $N \sim I^{\Gamma}$ are recursively enumerable in $g_{\Gamma}^{\#}$ and that from this we can conclude that $I^{\Gamma}$ is recursive in $g_{\Gamma}^{\#}$. We begin by reducing $I^{\Gamma}$ to $K^{\Gamma}$. 
LEMMA 5.6. If $\Gamma$ subsumes $U$, there exists a primitive recursive function $\tau$ such that $u \in I^{\Gamma} \leftrightarrow \tau(u) \in K^{\Gamma}$.

Proof. As usual we describe a construction for $\tau$ in terms of its own index and leave to the reader the proof by induction that $\tau$ has the required property. Set $\tau(\langle 0, m\rangle)=0$. Recall that

$$
\langle 1, u, a\rangle \in I^{\Gamma} \leftrightarrow u \in I^{\Gamma} \wedge \forall p\left(\{a\}^{[u ; \Gamma]}(p) \in I^{\Gamma}\right) .
$$

From the previous three lemmas it follows that for $u \in I^{\Gamma}$

Hence

$$
m \in[u ; \Gamma] \leftrightarrow \rho(\sigma(u), m, 0) \in K^{\Gamma}
$$

$$
\begin{aligned}
&\langle 1, u, a\rangle \in I^{\Gamma} \leftrightarrow u \in I^{\Gamma} \wedge \forall p \exists s \forall i_{i<\ln (s)}\left(\rho\left(\sigma(u), i,(s)_{i}\right) \in K^{\Gamma}\right. \\
&\left.\wedge T(s, a, p) \wedge U(\ln (s)) \in I^{\Gamma}\right) .
\end{aligned}
$$

Now an appropriate definition for $\tau$ can be found using the functions of 5.3. We could set $\tau(\langle 2, v\rangle)=\tau(v)$, but to simplify the right-to-left induction it is expedient to set $\tau(\langle 2, v\rangle)=\langle 1, b\rangle$ where $\forall x[\{b\}(x)=\tau(v)]$.

Our main tool in obtaining the result of this section is the ordinal comparison technique introduced by Gandy [Ga] and developed further by Moschovakis [Mo 1, 2]. We shall assign ordinals to members of $K^{\Gamma}$ in a slightly unorthodox way. Let $\mathscr{X}$ be any set (not necessarily of integers) and $F$ any function from $\mathscr{X}$ into the ordinals. Then we define

and

$$
\sup _{x \in \mathscr{X}}^{+} F(x)=\sup \{F(x)+1 / x \in \mathscr{X}\}
$$

$$
\inf _{x \in \mathscr{X}}^{+} F(x)=\inf \{F(x)+1 / x \in \mathscr{X}\} .
$$

If $u \notin K^{\Gamma}$, let $|u|^{\Gamma}=\Omega$. For $u \in K^{\Gamma}$ we assign ordinals recursively on the structure of $K^{\Gamma}$ :

$$
|\langle 1, a\rangle|^{\Gamma}=\inf _{\beta \in M}^{+} \sup _{y \in N}^{+}|\{a\}(\beta(y))|^{\Gamma} ; \quad|\langle 2, a\rangle|^{\Gamma}=\sup _{\beta \in M}^{+} \inf _{y \in N}^{+}|\{a\}(\beta(y))|^{\Gamma} ;
$$

and $\kappa^{\Gamma}=\sup \left\{|u| \Gamma / u \in K^{\Gamma}\right\}$.

For reference in the proof of the following theorem we note some easily derived formulas. All quantifiers, $\inf ^{+}$, and sup $^{+}$are over $\mathscr{X}$.

(A) inf $^{+} F \leqq$ inf $^{+} G \leftrightarrow \exists x \forall y(F(x) \leqq G(y))$;

(B) $\sup ^{+} F \leqq \sup ^{+} G \leftrightarrow \forall x \exists y(F(x) \leqq G(y))$;

(C) inf $^{+} G<$ inf $^{+} F \leftrightarrow \forall x \exists y(G(y)<F(x))$;

(D) $\sup ^{+} G<\sup ^{+} F \leftrightarrow \exists x \forall y(G(y)<F(x))$;

(E) $\sup ^{+} F \leqq \inf ^{+} G \leftrightarrow \forall x \forall y(F(x) \leqq G(y))$;

(F) inf $^{+} F \leqq \sup ^{+} G \leftrightarrow \exists x \exists y(F(x) \leqq G(y))$;

(G) inf $^{+} G<\sup ^{+} F \leftrightarrow \exists x \exists y(G(y)<F(x))$;

(H) $\sup ^{+} G<\inf ^{+} F \leftrightarrow \forall x \forall y(G(y)<F(x))$. 
THEOREM 5.7. There exists a partial recursive function $\eta$ such that for any $u$ and $v$,

(a) $u \in K^{\Gamma}$ and $|u|^{\Gamma} \leqq|v|^{\Gamma} \rightarrow \eta\left(g_{\Gamma}^{\#}, u, v\right) \cong 0$;

(b) $|v|^{\Gamma}<|u|^{\Gamma} \rightarrow \eta\left(g_{\Gamma}^{\#}, u, v\right) \cong 1$.

Proof. We define $\eta$ by cases as follows. As usual, the recursion theorem is needed to untangle the recursive definition. We shall omit $g_{\Gamma}^{\#}$ as an explicit argu-

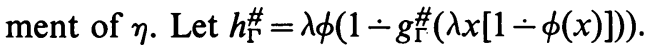

(1) $\eta(0, v) \cong 0$, for all $v$;

(2) $\eta(u+1,0) \cong 1$, for all $u$;

(3) $\eta(u+1, v+1) \cong 0$, if $v+1$ is not of the form $\langle 1, a\rangle$ or $\langle 2, a\rangle$ with $a \in \mathrm{PRI}$;

(4) $\eta(u+1,\langle 1, a\rangle) \cong \eta(u+1,\langle 2, a\rangle) \cong 1$, if $u+1$ is not of the form $\langle 1, b\rangle$ or $\langle 2, b\rangle$ with $b \in \mathrm{PRI}$;

(5) $\eta(\langle 1, a\rangle,\langle 1, b\rangle) \cong g_{\Gamma}^{\#}\left(\lambda m h_{\Gamma}^{\#}(\lambda n \eta(\{a\}(m),\{b\}(n)))\right)$;

(6) $\eta(\langle 2, a\rangle,\langle 1, b\rangle) \cong h_{\Gamma}^{\#}\left(\lambda m h_{\Gamma}^{\#}(\lambda n \eta(\{a\}(m),\{b\}(n)))\right)$;

(7) $\eta(\langle 1, a\rangle,\langle 2, b\rangle) \cong g_{\Gamma}^{\#}\left(\lambda m g_{\Gamma}^{\#}(\lambda n \eta(\{a\}(m),\{b\}(n)))\right)$;

(8) $\eta(\langle 2, a\rangle,\langle 2, b\rangle) \cong h_{\Gamma}^{\#}\left(\lambda m g_{\Gamma}^{\#}(\lambda n \eta(\{a\}(m),\{b\}(n)))\right)$.

We show that $\eta$ has the desired property by induction on $\min \left(|u|^{\Gamma},|v|^{\Gamma}\right)$. If any of cases (1)-(4) apply, the result is obvious. Suppose case (5) applies so $u=\langle 1, a\rangle$, $v=\langle 1, b\rangle$, and $a, b \in \mathrm{PRI}$.

First if $u \in K^{\Gamma}$ and $|u|^{\Gamma} \leqq|v|^{\Gamma}$, then

$$
\inf _{\alpha \in M}^{+} \sup _{x \in N}^{+}|\{a\}(\alpha(x))|^{\Gamma} \leqq \inf _{\beta \in M}^{+} \sup _{y \in N}^{+}|\{b\}(\beta(y))|^{\Gamma} .
$$

By (A) and (B) applied successively,

$$
\exists \alpha_{\alpha \in M} \forall \beta_{\beta \in M} \forall x \exists y\left[|\{a\}(\alpha(x))|^{\Gamma} \leqq|\{b\}(\beta(y))|^{\Gamma} \wedge\{a\}(\alpha(x)) \in K^{\Gamma}\right] .
$$

Then by the induction hypothesis and permutation of similar quantifiers,

$$
\exists \alpha_{\alpha \in M} \forall x \forall \beta_{\beta \in M} \exists y[\eta(\{a\}(\alpha(x)),\{b\}(\beta(y))) \cong 0],
$$

and thus $\eta(u, v) \cong 0$.

If, on the other hand, $|v|^{\Gamma}<|u|^{\Gamma}$, then using (C) and (D),

$$
\forall \alpha_{\alpha \in M} \exists \beta_{\beta \in M} \exists x \forall y\left[|\{b\}(\beta(y))|^{\Gamma}<|\{a\}(\alpha(x))|^{\Gamma}\right],
$$

so by the induction hypothesis and permutation of quantifiers,

$$
\forall \alpha_{\alpha \in M} \exists x \exists \beta_{\beta \in M} \forall y[\eta(\{a\}(\alpha(x)),\{b\}(\beta(y))) \cong 1],
$$

and it is easy to check that this implies $\eta(u, v) \cong 1$.

Suppose next that case (6) applies, so $u=\langle 2, a\rangle, v=\langle 1, b\rangle$, and $a, b \in$ PRI. If, first, $u \in K^{\Gamma}$ and $|u|^{\Gamma} \leqq|v|^{\Gamma}$, then

$$
\sup _{\alpha \in M}^{+} \inf _{x \in N}^{+}|\{a\}(\alpha(x))|^{\Gamma} \leqq \inf _{\beta \in M}^{+} \sup _{y \in N}^{+}|\{b\}(\beta(y))|^{\Gamma} .
$$

Applying first formula (E), we have

$$
\forall \alpha_{\alpha \in M} \forall \beta_{\beta \in M}\left[\inf _{x \in N}^{+}|\{a\}(\alpha(x))|^{\Gamma} \leqq \sup _{y \in N}^{+}|\{b\}(\beta(y))|^{\Gamma}\right]
$$


For any fixed $\alpha \in M$, let $x_{0}$ be chosen to minimize $\left|\{a\}\left(\alpha\left(x_{0}\right)\right)\right|^{\Gamma}$. Then

$$
\forall \beta_{\beta \in M}\left[\left|\{a\}\left(\alpha\left(x_{0}\right)\right)\right|^{\Gamma} \leqq \sup _{y \in N}^{+}|\{b\}(\beta(y))|^{\Gamma} \wedge\{a\}\left(\alpha\left(x_{0}\right)\right) \in K^{\Gamma}\right] .
$$

Then using formula (F),

$$
\forall \alpha_{\alpha \in M} \exists x \forall \beta_{\beta \in M} \exists y\left[|\{a\}(\alpha(x))|^{\Gamma} \leqq|\{b\}(\beta(y))|^{\Gamma} \wedge\{a\}(\alpha(x)) \in K^{\Gamma}\right],
$$

and from the definition of $\eta$ and the induction hypothesis, $\eta(u, v) \cong 0$.

If case (6) applies and $|v|^{\Gamma}<|u|^{\Gamma}$, then by (G) and (H),

$$
\exists \alpha_{\alpha \in M} \exists \beta_{\beta \in M} \forall x \forall y\left[|\{b\}(\beta(y))|^{\Gamma}<|\{a\}(\alpha(x))|^{\Gamma}\right] .
$$

Then the middle two quantifiers can be interchanged by a rule of logic and the same methods as above yield $\eta(u, v) \cong 1$.

The cases (7) and (8) are handled by the same straightforward techniques and we therefore omit the remainder of the proof.

COROLlARY 5.8. If $\Gamma$ subsumes $U$, then a necessary and sufficient condition that a set $A \subseteq N$ be recursive in $g_{\Gamma}^{\#}$ is that both $A$ and $N \sim A$ be recursively enumerable in $g_{\Gamma}^{\#}$.

Proof. That the condition is necessary is obvious. By 5.4 we may assume that for some $a$ and $b$,

$$
\begin{aligned}
A & =\left\{m /\{a\}\left(g_{\Gamma}^{\#}, m\right) \cong 0\right\}=\left\{m / \rho(a, m, 0) \in K^{\Gamma}\right\} \\
N \sim A & =\left\{m /\{b\}\left(g_{\Gamma}^{\#}, m\right) \cong 0\right\}=\left\{m / \rho(b, m, 0) \in K^{\Gamma}\right\} .
\end{aligned}
$$

With $\eta$ as in the preceding theorem, let

$$
\psi(m) \cong \eta\left(g_{\Gamma}^{\#}, \rho(a, m, 0), \rho(b, m, 0)\right) .
$$

Then

$$
\begin{aligned}
m \in A & \rightarrow \rho(a, m, 0) \in K^{\Gamma} \wedge \rho(b, m, 0) \notin K^{\Gamma} \\
& \rightarrow \rho(a, m, 0) \in K^{\Gamma} \wedge|\rho(a, m, 0)|^{\Gamma} \leqq|\rho(b, m, 0)|^{\Gamma} \\
& \rightarrow \psi(m) \cong 0 .
\end{aligned}
$$

Similarly, $m \in N \sim A \rightarrow \psi(m) \cong 1$. Thus $\psi$ is the characteristic function of $A$ and is clearly recursive in $g_{\Gamma}^{\#}$.

COROLlary 5.9. If $\Gamma$ subsumes $U$, then $K^{\Gamma}$ and $I^{\Gamma}$ are recursively enumerable in $g_{\Gamma}^{\#}$.

Proof. The result for $I^{\Gamma}$ follows from that for $K^{\Gamma}$ by 5.6. Let

$$
D(a) \leftrightarrow\{a\}\left(g_{\Gamma}^{\#}, a\right) \cong 0 \text { and } \rho_{1}(a)=\rho(a, a, 0)
$$

so $D(a) \leftrightarrow \rho_{1}(a) \in K^{\Gamma}$. Let $\nu=\sup \left\{\left|\rho_{1}(a)\right|^{\Gamma} / D(a)\right\}$. Suppose $\nu<\kappa^{\Gamma}$, so for some $u \in K^{\Gamma},|u|^{\Gamma}=\nu$. Then by Theorem 5.7 ,

$$
\begin{gathered}
D(a) \rightarrow \rho_{1}(a) \in K^{\Gamma} \wedge\left|\rho_{1}(a)\right|^{\Gamma} \leqq|u|^{\Gamma} \rightarrow \eta\left(g_{\Gamma}^{\#}, \rho_{1}(a), u\right) \cong 0 ; \\
\neg D(a) \rightarrow|u|^{\Gamma}<\left|\rho_{1}(a)\right|^{\Gamma}=\Omega \rightarrow \eta\left(g_{\Gamma}^{\#}, \rho_{1}(a), u\right) \cong 1 .
\end{gathered}
$$


Thus $D$ would be recursive in $g_{\Gamma}^{\#}$, a situation which is easily seen by a standard diagonal argument to be impossible. Hence $\nu=\kappa^{\Gamma}$ and

$$
K^{\Gamma}=\left\{u / \exists a\left[D(a) \wedge \eta\left(g_{\Gamma}^{\#}, u, \rho_{1}(a)\right) \cong 0\right]\right\}=\left\{u / \exists a\left[\varphi\left(g_{\Gamma}^{\#}, u, a\right) \cong 0\right]\right\}
$$

for an appropriate partial recursive $\varphi$. If $\alpha$ is the primitive recursive function by which $\Gamma$ subsumes $U$,

$$
K^{\Gamma}=\left\{u / g_{\Gamma}^{\#}\left(\lambda a \varphi\left(g_{\Gamma}^{\#}, u, \alpha(a)\right)\right) \cong 0\right\} .
$$

THEOREM 5.10. If $\Gamma$ subsumes $\mathscr{A}$, then $N \sim I^{\Gamma}$ is recursively enumerable in $g_{\Gamma}^{\#}$.

Proof. To each $u \in N$ we shall associate a sequence tree $T_{u}$, which is recursively enumerable in $g_{\Gamma}^{\#}$ and well-ordered just in case $u \in I^{\Gamma}$. Suppose this is done and $\varphi$ is a partial recursive function such that

$$
T_{u}(s) \leftrightarrow \varphi\left(g_{\Gamma}^{\#}, u, s\right) \cong 0 .
$$

Let $\alpha$ be the primitive recursive function by which $\Gamma$ subsumes $\mathscr{A}$. Then

$$
u \in N \sim I^{\Gamma} \leftrightarrow \exists \gamma \forall z T_{u}(\bar{\gamma}(z)) \leftrightarrow \exists \beta_{\beta \in M} \forall y T_{u}(\alpha(\beta(y))) \leftrightarrow g_{\Gamma}^{\#}\left(\lambda x \varphi\left(g_{\Gamma}^{\#}, u, \alpha(x)\right)\right) \cong 0 .
$$

Toward the construction of $T_{u}$, let $P_{1}(u, v)$ be the disjunction of (i)-(iv) below and $P_{2}(u, v, \delta)$ the disjunction of (v) and (vi).

For legibility we write $w$ for $(u)_{1}$ and $a$ for $(u)_{2}$.

(i) $u=\langle 0, w\rangle$ and $v=u$;

(ii) $u=\langle 1, w, a\rangle$ and $v=w$;

(iii) $u=\langle 2, w\rangle$ and $v=w$;

(iv) $u$ is of none of these forms and $v=0$;

(v) $u=\langle 1, w, a\rangle$ and $\operatorname{Dm}\left(\{a\}^{\delta}\right)=N$ and $\exists p\left[v=\{a\}^{\delta}(p)\right]$;

(vi) $u=\langle 1, w, a\rangle$ and $\operatorname{Dm}\left(\{a\}^{\delta}\right) \neq N$ and $v=0$.

Let $\sigma$ be the primitive recursive function of 5.5 and let $\psi_{w}=\lambda m\{\sigma(w)\}\left(g_{\Gamma}^{\#}, m\right)$. Set

$$
Q(u, v) \leftrightarrow P_{1}(u, v) \quad \text { or } \quad\left[\mathrm{Dm}\left(\psi_{w}\right)=N \quad \text { and } \quad P_{2}\left(u, v, \psi_{w}\right)\right] .
$$

Since $\Gamma$ subsumes $\mathscr{A}$ it also subsumes $U$, so by the method of proof of 5.2, $Q$ is recursively enumerable in $g_{\Gamma}^{\#}$. Finally,

$$
\begin{aligned}
T_{u}(s) \leftrightarrow & s=\varnothing \\
& \vee\left[(s)_{0}=u \wedge \forall i_{i<\ln (s)-1} Q\left((s)_{i},(s)_{i+1}\right) \wedge \forall m\left((s)_{\ln (s)-1} \neq\langle 0, m\rangle\right)\right] .
\end{aligned}
$$

Then $T_{u}$ is recursively enumerable in $g_{\Gamma}^{\#}$ and we claim

$$
u \in I^{\Gamma} \leftrightarrow \forall \gamma \exists z \neg T_{u}(\bar{\gamma}(z)) .
$$

The proof from left to right is a straightforward induction over $I^{\Gamma}$. Suppose first $u=\langle 0, m\rangle$. Then $\neg T_{u}(\bar{\gamma}(1))$ for any $\gamma$. Suppose $u=\langle 1, w, a\rangle \in I^{\Gamma}$ but for some $\gamma, \forall z T_{u}(\bar{\gamma}(z))$; that is, $\gamma(0)=u$ and $\forall z Q(\gamma(z), \gamma(z+1))$. Since $w \in I^{\Gamma}, \psi_{w}$ is the characteristic function of $[w ; \Gamma]$ and thus since $Q(u, \gamma(1)), \gamma(1) \in I^{\Gamma}$ and precedes $u$. 
Then $\gamma_{1}=\lambda x \gamma(x+1)$ is such that $\forall z T_{\gamma(1)}\left(\bar{\gamma}_{1}(z)\right)$, which contradicts the induction hypothesis. The case $u=\langle 2, w\rangle$ is similar but easier.

For the proof in the other direction we use bar induction in a form, which differs only slightly from that of [Kl-Ve, p. 52]: for any predicates $T$ and $S$, if

$$
\text { (a) } \forall \gamma \exists z \neg T(\bar{\gamma}(z)) \text {, }
$$

and for all $s, t$,
(b) $T(s) \wedge t \subseteq s \rightarrow T(t)$,
(c) $\neg T(s) \rightarrow S(s)$,
(d) $\forall v[S(s\langle v\rangle)] \rightarrow S(s)$,

then

$$
\text { (e) } \forall s[S(s)]
$$

We apply this with $T=T_{u}$ and $S=S_{u}$, where

$$
S_{u}(s) \leftrightarrow\left(\left[(s)_{0}=u \wedge \forall i_{i<\ln (s) \div 1} Q\left((s)_{i},(s)_{i+1}\right)\right] \rightarrow(s)_{\ln (s)-1} \in I^{\Gamma}\right) .
$$

It is easy to check (using (i)) that (b) and (c) hold for all $T_{u}$ and $S_{u}$. Assume for the moment that (d) does also. Then for any $u$ such that the right side of $\left(^{*}\right)$ holds, (a) is true of $T_{u}$ and we conclude $\forall s\left[S_{u}(s)\right]$. In particular, $S_{u}(\langle u\rangle)$ which implies $u \in I^{\Gamma}$.

To establish (d) for $S_{u}$, let $s=t\langle w\rangle$ and suppose $\forall v\left[S_{u}(s\langle v\rangle)\right],(s)_{0}=u$, and $\forall i_{i<\ln (s)-1} Q\left((s)_{i},(s)_{i+1}\right)$. From this it follows that for any $v, Q(w, v) \rightarrow v \in I^{\Gamma}$. It suffices to show $w \in I^{\Gamma}$. If $w=\langle 0, m\rangle$ we are done. Suppose $w=\langle 1, z, a\rangle$. Since by (ii), $P_{1}(w, z)$, hence $Q(w, z)$, so $z \in I^{\Gamma}$. Hence $\psi_{z}$ is the characteristic function of $[z ; \Gamma]$ and in particular $\operatorname{Dm}\left(\psi_{z}\right)=N$. If $\operatorname{Dm}\left(\{a\}^{\psi_{z}}\right) \neq N$, then by (vi), $P_{2}\left(w, 0, \psi_{z}\right)$, so $Q(w, 0)$ and $0 \in I^{\Gamma}$, which is false. Hence $\{a\}^{\psi_{z}}$ is a total function and by (v), for all $p, Q\left(w,\{a\}^{\psi} z(p)\right)$, so $\{a\}^{\psi} z(p) \in I^{\Gamma}$. Thus $w \in I^{\Gamma}$. The case $w=\langle 2, z\rangle$ is similar but easier. Finally $w$ must be one of these forms, as, if not, $Q(w, 0)$ by (iv) and hence again $0 \in I^{\Gamma}$.

Corollary 5.11. If $\Gamma$ subsumes $\mathscr{A}$ :

(a) $I^{\Gamma}$ is recursive in $g_{\Gamma}^{\#}$;

(b) ${ }_{1} \mathrm{sc}\left(g_{\Gamma}\right) \subset_{1} \mathrm{sc}\left(g_{\Gamma}^{\#}\right)$ and ${ }_{1} \mathrm{sc}\left(g_{\Gamma}\right) \neq{ }_{1} \mathrm{sc}\left(g_{\Gamma}^{\#}\right)$.

Proof. (a) is immediate from 5.8-5.10. (b) then follows from 5.5 and the remark following it.

6. Set derivatives and the $R$-operator. In this section we give a second characterization of sets of the form $R\left(\lambda s \Lambda_{P(s)}\right)(F)$ in terms of a set derivative based on $P$. A consequence is that each set of this form can be expressed as an $\Omega$-intersection of simpler sets. In the case $P(s)=\{\lambda x(p) / p \in N\}$ when $\Lambda_{P(s)}=U$ and $R\left(\lambda s \Lambda_{P(s)}\right)=\mathscr{A}$, the derivative is essentially the same as the ordinary topological derivative on $N^{N}$ under the Baire topology, and the corresponding representation is that of analytic sets as the intersection of their constituents (cf. [Kur, pp. 150-151], [Ba, II.D], and [Lu]). 
These results seem to be due to Ljapunov and can be found in quite a different form in [Lj 1].

DEFINITION 6.1. For any $P \in \mathscr{P}\left(N^{N}\right)^{N}$, any $S \subseteq N$, and any ordinal $\nu$ :

(a) $D_{P}(S)=\left\{s / s \in S \wedge \exists \beta_{\beta \in P(s)} \forall x(s\langle\beta(x)\rangle \in S)\right\}$;

(b) $D_{P}^{0}(S)=S, D_{P}^{(\nu)}(S)=\cap\left\{D_{P}^{\xi}(S) / \xi<\nu\right\}, D_{P}^{\nu}(S)=D_{P}\left(D_{P}^{(\nu)}(S)\right)$.

LEMMA 6.2. For any $P \in \mathscr{P}\left(N^{N}\right)^{N}$ and any $S, T \subseteq N$ :

(a) $D_{P}(S) \subseteq S$;

(b) $S \subseteq T \rightarrow D_{P}(S) \subseteq D_{P}(T)$;

(c) $\exists v_{v<\Omega} \forall \xi_{\xi>v}\left(D_{P}^{\xi}(S)=D_{P}^{v}(S)\right)$.

Proof. (a) and (b) are trivial. (c) follows simply from the denumerability of $S$ and the obvious fact that if ever $D_{P}^{\nu}(S)=D_{P}^{(\nu)}(S)$, then $D_{P}^{\xi}(S)=D_{P}^{v}(S)$ for all $\xi \geqq \nu$.

As in $\S 4$, when $P=\lambda s(M)$, we shall write $D_{M}$ and, if $M$ is the canonical base for $\Gamma$, also $D_{\Gamma}$ for $D_{\lambda s(M)}$.

LemmA 6.3. For any $P \in \mathscr{P}\left(N^{N}\right)^{N}$, any $S \subseteq N$, and any $s \in N$, let $P_{s}=\lambda t P(s * t)$. Then the following are equivalent:

(a) $s \in D_{P}^{(\Omega)}(S)$;

(b) $\{t / s * t \in S\}$ includes a $P_{s}$-fan;

(c) $\varnothing \in D_{P_{s}}^{(\Omega)}(\{t / s * t \in S\})$.

Proof. The equivalence of (b) and (c) follows from that of (a) and (b) via the substitutions of $\varnothing$ for $s, P_{s}$ for $P$, and $\{t / s * t \in S\}$ for $S$. Suppose first (a) $s \in D_{P}^{(\Omega)}(S)$. We shall define by induction sets $T_{m} \subseteq\left\{t / s * t \in D_{P}^{(\Omega)}(S)\right\}$ such that $T=\cup\left\{T_{m} / m \in N\right\}$ is a $P_{s}$-fan. Since $D_{P}^{(\Omega)}(S) \subseteq S$, this implies (b). Let $T_{0}=\varnothing$ and assume $T_{m}$ defined. By the previous lemma there exists an ordinal $\nu$ such that $D_{P}^{v}(S)=D_{P}^{(\Omega)}(S)$. Then by the induction hypothesis, $T_{m} \subseteq\left\{t / s * t \in D_{P}^{v+1}(S)\right\}$, that is,

$$
\forall t\left[t \in T_{m} \rightarrow \exists \beta_{\beta \in P_{s}(t)} \forall x\left(s * t\langle\beta(x)\rangle \in D_{P}^{v}(S)\right)\right] .
$$

For each $t \in T_{m}$, let $\beta_{t}$ be such a function (axiom of choice) and set

$$
T_{m+1}=\left\{t\left\langle\beta_{t}(x)\right\rangle / t \in T_{m} \wedge x \in N\right\} .
$$

Then $T_{m+1} \subseteq\left\{t / s * t \in D_{P}^{v}(S)\right\}=\left\{t / s * t \in D_{P}^{(\Omega)}(S)\right\}$. It is obvious that $T$ is a $P_{s}$-fan.

Conversely, suppose $\{t / s * t \in S\}$ includes a $P_{s}$-fan $T$. We show by transfinite induction that for all $\nu$

$$
T \subseteq\left\{t / s * t \in D_{P}^{v}(S)\right\} .
$$

Then since $\varnothing \in T$ it follows that $s \in D_{P}^{(\Omega)}(S)$. The case $\nu=0$ holds by hypothesis. Suppose $\nu>0$ and $t \in T$. There exists a $\beta \in P_{s}(t)$ such that $\forall x(t\langle\beta(x)\rangle \in T)$. By the induction hypothesis,

$$
\forall x\left(s * t\langle\beta(x)\rangle \in D_{P}^{(v)}(S)\right)
$$

and thus $s * t \in D_{P}^{v}(S)$. 
CoROllarY 6.4. For any $P \in \mathscr{P}\left(N^{N}\right)^{N}$ and any family $F$,

$$
R\left(\lambda s \Lambda_{P(s)}\right)(F)=\left\{m / \varnothing \in D_{P}^{(\Omega)}(\{t / m \in F(t)\})\right\} .
$$

Proof. Obvious.

The representation of $R\left(\lambda_{s} \Lambda_{P(s)}\right)(F)$ as an intersection follows immediately:

$$
R\left(\lambda s \Lambda_{P(s)}\right)(F)=\cap\left\{\left\{m / \varnothing \in D_{P}^{v}(\{t / m \in F(t)\})\right\} / \nu<\Omega\right\} .
$$

7. Culmination. We are now almost ready to put together results of the preceding sections to obtain our main result-that if $\Gamma$ subsumes $U$, then $\Delta_{1}^{R^{*} \Gamma}$ is the class of sets recursive in $g_{\Gamma}^{\#}$ and if $\Gamma$ subsumes $\mathscr{A}$, then $\Delta_{1}^{R \cdot \Gamma}$ properly includes $\mathscr{C}^{\Gamma}$. The intuitive content of our first lemma is that if $\Lambda_{M}$ subsumes $\Lambda_{P(s)}$ uniformly in $s$, then $\Lambda_{R M}$ subsumes $\Lambda_{R P}$.

Lemma 7.1. For any $P \in \mathscr{P}\left(N^{N}\right)^{N}$, any $M \subseteq N^{N}$, and any function $\pi$ such that for all $A \subseteq N$ and all $s$,

$$
\exists \beta_{\beta \in P(s)} \forall x(\beta(x) \in A) \leftrightarrow \exists \gamma_{y \in M} \forall y(\pi(s, \gamma(y)) \in A),
$$

there exists a function $\psi$ primitive recursive in $\pi$ such that for any $A$,

$$
\exists \beta_{\beta \in R P} \forall x(\beta(x) \in A) \leftrightarrow \exists \gamma_{\gamma \in R M} \forall y(\psi(\gamma(y)) \in A) .
$$

Proof. We define $\psi$ recursively from $\pi$ by the equations:

$$
\psi(\varnothing)=\varnothing, \quad \psi(t\langle n\rangle)=\psi(t)\langle\pi(\psi(t), n)\rangle .
$$

It will suffice to show:

(a) for any $P$-fan $S, \psi^{-1}(S)$ includes an $M$-fan;

(b) for any $M$-fan $T, \psi^{*}(T)$ is a $P$-fan.

The following chain of equivalences uses only the definition of $\pi$ :

$$
\begin{aligned}
\exists \alpha_{\alpha \in P(s)} \forall x(s\langle\alpha(x)\rangle \in S) & \leftrightarrow \exists \alpha_{\alpha \in P(s)} \forall x(\alpha(x) \in\{n / s\langle n\rangle \in S\}) \\
& \leftrightarrow \exists \delta_{\delta \in M} \forall y(\pi(s, \delta(y)) \in\{n \mid s\langle n\rangle \in S\}) \\
& \leftrightarrow \exists \delta_{\delta \in M} \forall y(s\langle\pi(s, \delta(y))\rangle \in S) .
\end{aligned}
$$

Suppose first that $S$ is a $P$-fan and let $T=\psi^{-1}(S)$. Since $\varnothing \in S$ and $\psi(\varnothing)=\varnothing$, also $\varnothing \in T$. Suppose $t \in T$ and $s=\psi(t) \in S$. Since $S$ is a $P$-fan,

$$
\exists \alpha_{\alpha \in P(s)} \forall x(s\langle\alpha(x)\rangle \in S),
$$

and by the equivalence above,

$$
\exists \delta_{\delta \in M} \forall y(s\langle\pi(s, \delta(y))\rangle \in S),
$$

hence $\exists \delta_{\delta \in M} \forall y(\psi(t\langle\delta(y)\rangle) \in S)$, and $\exists \delta_{\delta \in M} \forall y(t\langle\delta(y)\rangle \in T)$. Thus $T$ includes an $M$-fan.

Conversely, if $T$ is an $M$-fan and $S=\psi^{*}(T)$, it is easy to check (and we leave it to the diligent reader to do so) that $\varnothing \in S$ and $s_{1} \subseteq s_{2} \in S \rightarrow s_{1} \in S$. The argument that $S$ satisfies condition (iii) proceeds in reverse order up the above chain of statements. 
Now let $\Gamma$ be a fixed positive analytic operation with canonical base $M$. Let

$$
M^{*}=\left\{\gamma / \exists \alpha_{\alpha \in M} \forall x \forall \beta_{\beta \in M} \exists y[\langle\alpha(x), \beta(y)\rangle \in \text { range } \gamma]\right\} .
$$

Referring to 3.7 and the definition of $\Gamma^{*}$ following 4.6 , it is immediate that $M^{*}$ is the canonical base for $\Gamma^{*}$.

LEMMA 7.2. There exists a $P \in \mathscr{P}\left(N^{N}\right)^{N}$, a recursive function $\pi$, and a recursive set $B$ such that for any $s, t, u$, and any $A \subseteq N$ :

(a) $u \in K^{\Gamma} \leftrightarrow t\langle u\rangle \notin D_{P}^{(\Omega)}(B)$;

(b) $\exists \delta_{\delta \in P(s)} \forall z[\delta(z) \in A] \leftrightarrow \exists \gamma_{\gamma \in M^{*}} \forall z[\pi(s, \gamma(z)) \in A]$.

Proof. Let $K_{v}^{\Gamma}$ denote the part of $K^{\Gamma}$ constructed by the $\nu$ th stage and $K_{(v)}^{\Gamma}$ $=\cup\left\{K_{\xi}^{\Gamma} / \xi<\nu\right\}$. We shall define $P, \pi$, and $B$ to satisfy (b) and prove by induction on $v$ that for all $t$ and $u$,

$$
u \in K_{v}^{\Gamma} \leftrightarrow t\langle u\rangle \notin D_{P}^{v}(B)
$$

Let

$$
\begin{aligned}
\pi(s, w) & =\{a\}\left((w)_{1}\right), & & \text { if } s=t\langle\langle 1, a\rangle\rangle \wedge a \in \mathrm{PRI} ; \\
& =\{a\}\left((w)_{0}\right), & & \text { if } s=t\langle\langle 2, a\rangle\rangle \wedge a \in \mathrm{PRI} ; \\
& =1, & & \text { otherwise. }
\end{aligned}
$$

For each $s, P(s)=\left\{\lambda z \pi(s, \gamma(z)) / \gamma \in M^{*}\right\}$.

Then (b) is automatically satisfied. Let $B=\{s / \forall t(s \neq t\langle 0\rangle)\}$, so (c) is satisfied for $\nu=0$. Suppose $\nu>0$ and assume as induction hypothesis that for all $t$ and $u$,

$$
u \in K_{(v)}^{\Gamma} \leftrightarrow t\langle u\rangle \notin D_{P}^{(v)}(B) .
$$

Suppose first $u=\langle 1, a\rangle$ with $a \in \mathrm{PRI}$ and $s=t\langle u\rangle$. Then,

$$
\begin{aligned}
u \in K_{v}^{\Gamma} & \leftrightarrow \exists \beta_{\beta \in M} \forall y\left[\{a\}(\beta(y)) \in K_{(v)}^{\Gamma}\right] \\
& \leftrightarrow \neg \forall \beta_{\beta \in M} \exists y\left[\{a\}(\beta(y)) \notin K_{(v)}^{\Gamma}\right] \\
& \leftrightarrow \neg \exists \gamma_{\gamma \in M^{*}} \forall z\left[\{a\}\left((\gamma(z))_{1}\right) \notin K_{(v)}^{\Gamma}\right] \\
& \leftrightarrow \neg \exists \gamma_{\gamma \in M^{*}} \forall z\left[\pi(s, \gamma(z)) \notin K_{(v)}^{\Gamma}\right] \\
& \leftrightarrow \neg \exists \delta_{\delta \in P(s)} \forall z\left[\delta(z) \notin K_{(v)}^{\Gamma}\right] \\
& \leftrightarrow \neg \exists \delta_{\delta \in P(s)} \forall z\left[s\langle\delta(z)\rangle \in D_{P}^{(v)}(B)\right] \\
& \leftrightarrow s \notin D_{P}^{v}(B) .
\end{aligned}
$$

The proof for $u=\langle 2, a\rangle$ is almost identical. If $u$ is of neither of these forms and $s=t\langle u\rangle$, then $P(s)=\{\lambda x \cdot 1\}$. Since $1 \notin K_{(v)}^{\Gamma}, s\langle 1\rangle \in D_{P}^{(v)}(B)$, hence

$$
\exists \delta_{\delta \in P(s)} \forall z\left[s\langle\delta(z)\rangle \in D_{P}^{(v)}(B)\right] .
$$

Therefore $s \notin D_{P}^{v}(B) \leftrightarrow s \notin D_{P}^{(v)} \leftrightarrow u \in K_{(v)}^{\Gamma} \leftrightarrow u \in K_{v}^{\Gamma}$, as these are all true just in case $u=0$.

THEOREM 7.3. $K^{\Gamma} \in \Pi_{1}^{R^{*} \Gamma}$.

Proof. Let $B, P$, and $\pi$ be as in the preceding lemma, and for each $u$ set $P_{u}$ $=\lambda s P(\langle u\rangle s)$. Then by Lemmas 7.2 and 6.3,

$$
u \in K^{\Gamma} \leftrightarrow\langle u\rangle \notin D_{P}^{(\Omega)}(B) \leftrightarrow \neg \exists \delta_{\delta \in R P_{u}} \forall z[\langle u\rangle \delta(z) \in B]
$$


Define $\psi$ as in the proof of 7.1, but uniformly for the families $P_{u}$ :

$$
\psi(u, \varnothing)=\varnothing, \quad \psi(u, t\langle n\rangle)=\psi(u, t)\langle\pi(\langle u\rangle \psi(u, t), n)\rangle .
$$

Then by 7.2 , for any $s, u$ and $A$,

$$
\exists \delta_{\delta \in P_{u}(s)} \forall z[\delta(z) \in A] \leftrightarrow \exists \gamma_{\gamma \in M^{*}} \forall z[\pi(\langle u\rangle s, \gamma(z)) \in A] .
$$

Thus if $F(p)=\{u \mid\langle u\rangle \psi(u, p) \in B\}$,

$$
K^{\Gamma}=N \sim R^{*} \Gamma(F) \in \Pi_{1}^{R^{*} \Gamma} .
$$

THEOREM 7.4. If $\Gamma$ subsumes $U$, then for any $A \subseteq N, A \in \Pi_{1}^{R^{*} \Gamma} \rightarrow A$ is recursively enumerable in $g_{\Gamma}^{\#}$.

Proof. Let $F$ be a primitive recursive family such that $A=N \sim R^{*} \Gamma(F)$, and set

$$
L=\left\{\langle m, s\rangle / s \in D_{M^{\prime}}^{(S)}(\{t / m \in F(t)\})\right\} .
$$

It is easy to check from the definition of $D_{M^{*}}$ (6.1) that $L$ is the set defined inductively by the clauses:

(i) $m \notin F(s) \rightarrow\langle m, s\rangle \in L$;

(ii) $\forall \gamma_{\gamma \in M} \cdot \exists z[\langle m, s\langle\gamma(z)\rangle\rangle \in L] \rightarrow\langle m, s\rangle \in L$.

Since by $6.4, m \in A \leftrightarrow\langle m, \varnothing\rangle \in L$, it suffices by 5.9 to define a primitive recursive function $\rho$ such that $\langle m, s\rangle \in L \leftrightarrow \rho(m, s) \in K^{\Gamma}$. Using the recursion theorem, set $\rho(m, s)=0$ if $m \notin F(s)$, otherwise $\rho(m, s)=\langle 2, b\rangle$, where $b$ is a primitive recursive index such that $\{b\}(u)=\left\langle 1, a_{u}\right\rangle$ and $a_{u}$ is a primitive recursive index such that $\left\{a_{u}\right\}(v)=\rho(m, s\langle\langle u, v\rangle\rangle)$.

The basis of the proof by induction that $\rho$ is as required is obvious. The induction step is essentially the same in both directions:

$$
\begin{aligned}
\rho(m, s) \in K^{\Gamma} & \leftrightarrow \forall \alpha_{\alpha \in M} \exists x\left[\left\langle 1, a_{\alpha(x)}\right\rangle \in K^{\Gamma}\right] \\
& \leftrightarrow \forall \alpha_{\alpha \in M} \exists x \exists \beta_{\beta \in M} \forall y\left[\rho\left(m, s\langle\langle\alpha(x), \beta(y)\rangle) \in K^{\Gamma}\right]\right. \\
& \leftrightarrow \forall \gamma_{\gamma \in M} \cdot \exists z\left[\rho(m, s\langle\gamma(z)\rangle) \in K^{\Gamma}\right] \\
& \leftrightarrow \forall \gamma_{\gamma \in M} \cdot \exists z[\langle m, s\langle\gamma(z)\rangle\rangle \in L] \\
& \leftrightarrow\langle m, s\rangle \in L .
\end{aligned}
$$

COROLlary 7.5. If $\Gamma$ subsumes $U$, then for any $A \subseteq N$,

(a) $A \in \Pi_{1}^{R^{*} \Gamma} \leftrightarrow A$ is recursively enumerable in $g_{\Gamma}^{\# \text {; }}$

(b) $A \in \Delta_{1}^{R^{*} \Gamma} \leftrightarrow A$ is recursive in $g_{\Gamma}^{\#}$.

Proof. (a) is immediate from 5.4, 7.3, and 7.4. Then (b) follows from (a) by 5.8.

Corollary 7.6. If $\Gamma$ subsumes $\mathscr{A}$, then

$$
\mathscr{C} \Gamma \subset \Delta_{1}^{R^{*} \Gamma} \text { and } \quad \mathscr{C}^{\Gamma} \neq \Delta_{1}^{R^{*} \Gamma} .
$$

Proof. $\mathscr{C} \Gamma={ }_{1} \mathrm{sc}\left(g_{\Gamma}\right) \subset_{1} \mathrm{sc}\left(g_{\Gamma}^{\#}\right)=\Delta_{1}^{R^{*} \Gamma}$ by 2.10 and 7.5. The inclusion is strict by 5.11 .

8. On sets of functions. As we indicated in $\S 1$, one point of departure for the research reported in this paper was Addison's study of the effective Borel hierarchy. Addison's definition of the hierarchy differs from our 2.1, specialized to $\Gamma=U$, 
mainly in that $U$ is applied only to (absolutely) recursively enumerated families. Thus the set of indices is independent of the kind of sets indexed, so that effective Borel hierarchies over $N, N^{N}$, or any other separable space may be obtained by assigning sets of the corresponding basis to the indices $\langle 0, m\rangle$. In fact, Addison's primary concern was with the hierarchy over $N^{N}$.

Unfortunately, for operations $\Gamma$ more powerful than $U$, recursive enumeration does not suffice to generate all of ${ }_{1} \mathrm{sc}\left(g_{\Gamma}\right)$ and we were forced to adopt the more complicated schema 2.1. Although this definition no longer makes sense over an arbitrary space, since we need a theory of recursion relative to subsets of the space, it can be applied to $N^{N}$ by assigning to $\langle 0, m\rangle$ the set

$$
\left\{\alpha / \forall i_{i<\ln (m)}\left(\alpha(i)=(m)_{i}\right)\right\} .
$$

The resulting hierarchy has, however, some rather unnatural aspects. First, among the sets generated at least by the second level is $\Gamma(\lambda p\{\alpha / \alpha(p)=0\})$, whose characteristic function is exactly $g_{\Gamma}$. Since certainly the scope of the hierarchy is included in ${ }_{2} \mathrm{sc}\left(g_{\Gamma}\right)$, all enumerating functions are already available by the third level, and we might just as well admit from the beginning all enumerating functions recursive in $g_{\Gamma}$. This does not imply that the hierarchy is degenerate. Indeed, because of [Ad 3, p. 133] it probably is not. Still, one feels that arbitrary recursions in $g_{\Gamma}$, even if only with free number variables, are out of place in the early stages of a hierarchy for ${ }_{2} \mathrm{sc}\left(g_{\Gamma}\right)$. We considered in [Hi] a version not subject to this criticism: at level $v$ of the hierarchy over $N^{N}$ we admit enumerations recursive in sets which appear at some level $\xi, \xi<\nu$, of the hierarchy over $N$. Both of these constructions have, however, a more serious fault: if $\Gamma$ subsumes $\mathscr{A}$, they comprehend only a proper subset of ${ }_{2} \mathrm{sc}\left(g_{\Gamma}\right)$ (cf. [Hi, III.43]).

Another possibility for an effective $\Gamma$-hierarchy over $N^{N}$ is suggested by Moschovakis' definition of a hyperanalytic hierarchy [Mo 1]. For each $a \in N^{N}$ define $I^{\Gamma}(\alpha)$, and for each $u \in I^{\Gamma}(\alpha)$ a set $[u ; \Gamma, \alpha] \subseteq N$, just as in 2.1 except that enumerations are relative to $\alpha$ as well as to some previously constructed set. Then for any ordinal $\nu$ which is (the order-type of a well-ordering of $N$ ) recursive in $g_{\Gamma}$,

$$
[u ; \Gamma]_{v}^{2}=\left\{\alpha / u \in I_{(v)}^{\Gamma}(\alpha) \wedge 0 \in[u ; \Gamma, \alpha]\right\}
$$

is easily seen to be recursive in $g_{\Gamma}$. We do not know if such sets exhaust ${ }_{2} \mathrm{sc}\left(g_{\Gamma}\right)$, but we conjecture that they do. While possibly of interest as an alternative to Moschovakis" "skeletal" hierarchies, this construction seems too remote from its classical model to be considered its effective analogue.

Although there may be no natural extension of Theorem 2.10 to sets of functions, Corollary 7.5 can easily be extended to this case, and we sketch briefly how this is done. For each $\alpha \in N^{N}$, let $K^{\Gamma}(\alpha)$ be the set defined as in 5.2 with " $\{a\}$ " replaced by " $\{a\}^{\alpha}$ " and other appropriate notational changes, and set

$$
\mathscr{K}^{\Gamma}=\left\{\langle u, \alpha\rangle \mid u \in K^{\Gamma}(\alpha)\right\} .
$$


Theorems 5.4 and 5.7 can be uniformly relativized to $\alpha$ to yield functions $\rho$ and $\eta$ such that:

$$
\begin{aligned}
&\{a\}\left(g_{\Gamma}^{\#}, \alpha, s\right) \cong n \leftrightarrow \rho(a, s, n) \in K^{\Gamma}(\alpha) \\
& u \in K^{\Gamma}(\alpha) \wedge|u|_{\alpha}^{\Gamma} \leqq|v|_{\alpha}^{\Gamma} \rightarrow \eta\left(g_{\Gamma}^{\#}, \alpha, u, v\right) \cong 0, \\
&|v|_{\alpha}^{\Gamma}<|u|_{\alpha}^{\Gamma} \rightarrow \eta\left(g_{\Gamma}^{\#}, \alpha, u, v\right) \cong 1 .
\end{aligned}
$$

Essentially nothing new is needed for 5.7.1, and in proving 5.4.1 there is one additional case to consider:

$(a)_{0}=7:\{a\}\left(g_{\Gamma}^{\#}, \alpha,\langle m\rangle s\right) \cong \alpha(m)$. Choose $b \in$ PRI such that $\{b\}^{\alpha}(x)=(0$, if $\alpha(m)$ $=n ; 1$, otherwise $)$ and set $\rho(a,\langle m\rangle s, n)=\pi_{\exists}(b)$.

Then 5.8 holds for $A \subseteq N^{N}$ and we can prove similarly

$$
\mathscr{K}^{\Gamma} \text { is recursively enumerable in } g_{\Gamma}^{\#} \text {. }
$$

In the proofs of $7.2-3$, define $\pi^{\alpha}(s, w)$ by replacing " $\{a\}$ " by " $\{a\}^{\alpha}$ ", and $\psi^{\alpha}$ and $P^{\alpha}$ from $\pi^{\alpha}$ as before. Then

$$
\begin{aligned}
\langle u, \alpha\rangle \in \mathscr{K}^{\Gamma} & \leftrightarrow u \in K^{\Gamma}(\alpha) \leftrightarrow\langle u\rangle \notin D_{P^{\alpha}}^{(\Omega)}(B) \\
& \leftrightarrow \neg \exists \gamma_{y \in R M} \cdot \forall z\left[\langle u\rangle \psi^{\alpha}(u, \gamma(z)) \in B\right],
\end{aligned}
$$

which implies that $\mathscr{K}^{\Gamma}$ is definable in $\Pi_{1}^{R^{*} \Gamma}$ form. The extended version of 7.4 follows easily from 5.9.1 and hence we have 7.5 for all $A \subseteq N^{N}$.

A corollary is that for $\Gamma$ which subsume $U, g_{R^{*} \Gamma}$ is of the same degree as

$$
\lambda e \alpha\left[\{e\}\left(g_{\Gamma}^{\#}, \alpha\right) \downarrow\right],
$$

the superjump of $g_{\Gamma}^{\#}$. We would like to know (i) is $g_{R}$ of the same degree as the superjump of any (nonextended) functional $f$ ? (ii) Does there exist an operation $\Lambda$ such that $g_{\Lambda}$ is of the same degree as the superjump of $g_{\Gamma}$ ? If, as we believe, the answer to (i) is no, then Friedberg's theorem [Sa, p. 77], that a ${ }_{1}$ degree $d$ is the (ordinary) jump of another just in case $\boldsymbol{d} \geqq \boldsymbol{0}^{\prime}$, does not extend to type- 2 .

\section{BIBLIOGRAPHY}

[Ad 1] J. W. Addison, On some points of the theory of recursive functions, Ph.D. dissertation, Univ. of Wisconsin, Madison, 1954.

[Ad 2] - Analogies in the Borel, Lusin, and Kleene hierarchies. I and II, Abstracts 139 and 341, Bull. Amer. Math. Soc. 61 (1955), 75, 171-172.

[Ad 3] - Separation principles in the hierarchies of classical and effective descriptive set theory, Fund. Math. 46 (1959), 123-135.

[Ad 4] - "The theory of hierarchies," in Logic, methodology and philosophy of science, Proc. 1960 Internat. Congr. edited by Ernest Nagel, Patrick Suppes, and Alfred Tarski, Stanford Univ. Press, Stanford, California, 1962, pp. 26-37.

[Ad 5] - "Some problems in hierarchy theory," in Recursive function theory, Proc. 1961 Sympos., edited by J. C. E. Dekker, Proc. Sympos. Pure Math., Vol. 5, Amer. Math. Soc., Providence, R. I., 1962, pp. 123-130

[Ad 6] -, "The method of alternating chains," in Theory of models, Proc. 1963 Internat. Sympos. at Berkeley, edited by J. W. Addison, Leon Henkin and Alfred Tarski, North-Holland, Amsterdam, 1966, pp. 1-16. 
[Ad-Kl] J. W. Addison and S. C. Kleene, A note on function quantification, Proc. Amer. Math. Soc. 8 (1957), 1002-1006.

[Ba] R. F. Barnes, The classification of the closed-open and the recursive sets of numbertheoretic functions, Ph.D. dissertation, Univ. of California, Berkeley, 1965.

[Be] G. M. Benson, The theory of definability in applied predicate languages for number theory, Ph.D. dissertation, Univ. of California, Berkeley, 1966.

[Bo] E. Borel, Le calcul des intégrales définis, J. Math. Pures Appl. 8 (1912), 159-210.

[Ga] R. O. Gandy, General recursive functionals of finite type and hierarchies of functions, mimeographed copy of a paper given at the symposium on Mathematical Logic held at the University of Clermont-Ferrand, June, 1962. Similar material will appear in the Proceedings of the Summer School in Mathematical Logic held at the University of Leicester, August, 1964.

[Ha] F. Hausdorff, Set theory, Chelsea, New York, 1962, 352 pp.

[Hi] P. G. Hinman, Ad astra per aspera: hierarchy schemata in recursive function theory, Ph.D. dissertation, Univ. of California, Berkeley, 1966.

[Ka-Li] L. Kantorovitch and E. Livenson, Memoir on the analytical operations and projective sets. I and II, Fund. Math. 18 (1932), 214-279; 20 (1933), 54-97.

[Kl 1] S. C. Kleene, Introduction to metamathematics, Van Nostrand, New York, 1952, $\mathrm{x}+550 \mathrm{pp}$.

[KI 2] - Hierarchies of number-theoretic predicates, Bull. Amer. Math. Soc. 61 (1955), 193-213.

[Kl 3] - Recursive functionals and quantifiers of finite types. I and II, Trans. Amer. Math. Soc. 91 (1959), 1-52; 108 (1963), 106-142.

[Kl-Ve] S. C. Kleene and R. E. Vesley, The foundations of intuitionistic mathematics, NorthHolland, Amsterdam, 1965, vi 206 pp.

[Ko] A. N. Kolmogorov, Operations on sets, Mat. Sb. 35 (1928), 414-422. (Russian)

[Ku] K. Kunugui, Sur un théorème d'existence dans la théorie des ensembles projectifs, Fund. Math. 29 (1937), 167-181.

[Kur] K. Kuratowski, Topologie, 4th ed., Vol. 1, PWN, Warsaw, 1958, xii +494 pp.

[Le] H. Lebesgue, Sur les fonctions representables analytiquement, J. Math. Pures Appl. (6) 1 (1905), 139-216.

[Lj 1] A. A. Ljapunov, R-sets, Trudy Mat. Inst. Steklov. 40 (1953). (Russian)

[Lj 2] - On the classification of R-sets, Mat. Sb. 74 (1953), 255-262. (Russian)

[Lu] N. N. Luzin, Leçons sur les ensembles analytiques, Gauthier-Villars, Paris, 1930, xvi + 238 pp.

[Mo 1] Y. N. Moschovakis, Hyperanalytic predicates, Trans. Amer. Math. Soc. 129 (1967), 249-282.

[Mo 2] - Abstract first-order computability. I and II, Trans. Amer. Math. Soc. 138 (1969), 427-464, 465-504.

[Sa] G. E. Sacks, Degrees of unsolvability, Ann. of Math. Studies No. 55, Princeton Univ. Press, Princeton, N. J., 1963, xi 174 pp.

[Se] E. Selivanovskij, On a class of effective sets (sets C), Mat. Sb. 35 (1928), 379-413.

[Su] M. Suslin, Sur une définition des ensembles mesurables B sans nombres transfinis, C. R. Acad. Sci. Paris 164 (1917), 88-91.

UNIVERSITY OF MICHIGAN, ANn Arbor, Michigan 